\title{
TCS-CAIN: a deep multi-colour NIR survey of the Galactic plane ${ }^{\star}$
}

\author{
A. Cabrera-Lavers ${ }^{1,2}$, F. Garzón ${ }^{1,3}$, P. L. Hammersley ${ }^{1}$, B. Vicente ${ }^{1}$, and C. González-Fernández ${ }^{1}$ \\ 1 Instituto de Astrofísica de Canarias, 38200 La Laguna, Tenerife, Spain \\ e-mail: acabrera@gtc.iac.es \\ 2 GRANTECAN S.A., 38200 La Laguna, Tenerife, Spain \\ 3 Departamento de Astrofísica, Universidad de La Laguna, 38200 La Laguna, Tenerife, Spain
}

Received 18 October 2004 / Accepted 21 February 2006

\section{ABSTRACT}

\begin{abstract}
We describe a deep multi-colour NIR survey (TCS-CAIN) that has been recently completed at the Instituto de Astrofísica de Canarias (Spain). The survey is of selected areas distributed along the Galactic plane and it goes deeper than 2MASS or DENIS. Its aim was to explore the large-scale structure of the Milky Way and the Galactic components, in particular the Galactic bar. This survey has $\sim 10$ million point-source detections in $J, H$, and $K_{\mathrm{s}}$ filters with a photometric accuracy of $\sim 0.1$ mag in the three bands and a positional accuracy of $\sim 0.2^{\prime \prime}$ (based on the 2MASS catalogue as the astrometric reference). Approximately 530 fields have been observed in near plane regions of the Galaxy covering a total area of $\sim 41 \mathrm{deg}^{2}$ in the sky. This survey is at least one magnitude deeper than $2 \mathrm{MASS}$, and even more in the inner Galaxy where it is less limited by source confusion.
\end{abstract}

Key words. Galaxy: structure - infrared: stars

\section{Introduction}

Star counts have long been used to examine the stellar contents in the Galaxy (see Paul 1993; Hammersley et al. 1994, 1995; López-Corredoira et al. 2000, 2004, 2005); however, the structural parameters of the various morphological components are still not well determined. Reviews of star-count surveys can be found in Bahcall (1986), Majewski (1993), and Reid (1993). A large number of the visible studies have been directed towards the Galactic poles, where the extinction in the Galactic plane (GP) can be largely ignored. Star counts, velocity distributions, and metallicity have been used to determine and separate the various Galactic populations, such as the old disk, young disk, and halo. Detailed reviews of this work are given in a number of papers such as Gilmore et al. (1989), and Freeman (1987) and references therein. Hence, the vertical structure of the local Galaxy is well measured (although there still remain contentious issues such as the thick disk). The horizontal structures have been studied less well. Robin et al. (1992) have obtained deep visible star counts in the anticentre region and concluded that the disk has a scale length of $2.5 \mathrm{kpc}$ and a cutoff at about $14 \mathrm{kpc}$. However, apart from individual clear windows, visible star counts in the plane are limited to a maximum heliocentric distance of about $3 \mathrm{kpc}$ (Schmidt-Kaler 1977), because of the high extinction. A method for overcoming this, at least in part, is to observe in the near infrared (NIR) where the extinction is significantly lower while the detected flux is still largely dominated by stellar emission. The infrared absolute magnitude of most giants stars is sufficiently bright to allow them to be easily detected deep into the Galactic plane, whilst the range of intrinsic $J-K$ (or $J-H$ ) colour is sufficient to separate them on a colour-magnitude diagram. As well as being able to detect sources to a greater

* Catalogue is only available in electronic form at the CDS via anonymous ftp to cdsarc.u-strasbg.fr $(130.79 .128 .5)$ or via http://cdsweb.u-strasbg.fr/cgi-bin/qcat? J/A+A/453/371 distance, the infrared source counts are far less affected by local dust, and so the measured distribution of sources is closer to the true distribution. In the past two decades, there have been large advances in this topic with the use of detailed models of stellar distribution (Wainscoat et al. 1992; Bissantz \& Gerhard 2002; Robin et al. 2003) along with large area, high sensitivity and multi-colour star counts surveys.

The first large area NIR star count survey with sufficient depth to reach the inner Galaxy is the Two Micron Galactic Survey (TMGS, Garzón et al. 1993; Hammersley et al. 1999). This was a collaborative project between the Instituto de Astrofísica de Canarias (IAC), Tenerife, and Imperial College, London, to map large parts of the Galactic bulge and plane. Observations were made on the 1.5-m Carlos Sánchez Telescope (TCS) of the Observatorio del Teide, Tenerife, and some $300 \mathrm{deg}^{2}$ of sky have been mapped down to about $+10 \mathrm{mag}$ in $K$ with about 750000 sources being detected. The main areas are in strips about $30^{\circ}$ in RA (centred on the GP) by about $1.5^{\circ}$ in declination and they cover positive longitudes between the GC and the anticentre. Most TMGS sources lie within a few degrees of the GP, the majority having no optical counterparts to at least $m_{V}=+16$, implying that they are sources deep within the Galaxy. The observations ended in 1996, and the final catalogue was produced soon after this.

The NIR surveys (Garzón et al. 1993; Skrutskie et al. 1997; Epchtein et al. 1999) are extremely valuable tools for exploring the Galactic structure, as there is less interstellar extinction when compared with the optical bands. This allows the highly obscured in plane areas of the inner Galaxy to be observed. A full coverage of the sky is provided by the All Sky Release of the 2MASS survey (Cutri et al. 2003, http://www.ipac. caltech.edu/2mass). However, the 2MASS survey is strongly affected by source confusion very close to the GP, and in the inner Galaxy the effective limiting magnitude is 2 , or more, magnitudes brighter than the 
nominal values for the survey. For this reason the TCS-CAIN survey described here has concentrated on those regions where it will compliment the existing large area surveys like 2MASS or DENIS by offering better sensitivity for crowded areas. This is of particular interest in the inner Galaxy where this survey is expected to contribute to addressing many of the current ambiguities in the understanding of the structure of the Galaxy.

We wish to examine the morphology and luminosity functions of the various Galactic components; the disk, spiral arms, ring/bar, and bulge, as well as the local spur and molecular clouds. Our objective is to isolate each Galactic component where there is least contamination from the other components. When examining the inner Galaxy, it is very important that the other structures in the line-of-sight can be accurately accounted for (e.g. disk contamination of bulge counts). To this end the survey is composed of two types of observations: roughly $70 \%$ of the selected areas, mostly concentrated along the GP $\left(|b| \leq 5^{\circ}\right)$, produce a sparse map homogeneously covering the part of the GP visible from the Observatorio del Teide (OT); the remainder increase the sampling density for specific areas of interest, e.g. the bulge.

\section{The CAIN camera}

CAIN (CAmara INfrarroja) is a common user instrument at the $1.5 \mathrm{~m}$ TCS (Tenerife). It is a fully cryogenic (working at LN2 temperature) NIR camera developed at the IAC in several stages. In its final form, CAIN is an NIR imager with two plate-scale optics and equipped with a $256 \times 256$ pixel NICMOS 3 detector.

The instrument is contained in a single cryostat, whose entrance window acts as a field lens. For the survey the wide-field camera was used, which has 4 lenses and gives a plate scale of $1 \operatorname{arcsec} \mathrm{pix}^{-1}$. Hence the field of view (FOV) is $4.25 \times 4 ! 25$. The filter wheel mounts 6 broad and narrow band NIR filters, plus pupil optics, for alignment purposes, and a blocked position. The optical path is fully baffled and all of the cold mechanical parts near the optical path are painted in Nextel to minimise background contamination.

The CAIN detector is a Rockwell NICMOS 3 FPA mounted onto a dedicated fan-out board that connects to the clocking and control signals. There is a preamplifier external to the cryostat to buffer the signal. The detector controller is based on the SDSU architecture, which permits both destructive and non-destructive read-outs. This electronics has a fixed read-out time and reset time of $50 \mathrm{~ms}$ for the whole array, which corresponds to a pixel rate of $330 \mathrm{k}$ pixels per second on each of the four readout channels.

The system allows a number of different read-out modes: reset-read, correlated double sample (CDS), Fowler (multiple reads at the beginning and end of each integration), and ramp (equally spaced reads during the integration period). We used the Fowler mode during this project. Conceptually this is equivalent to CDS, in which the detector is read immediately after the reset, and the detector is left to accumulate photons during the given interval and then read again. The final signal is the difference between the two reads, and in this way, the fluctuations in the reset level can be eliminated. The Fowler read-out mode extends this by having multiple non-destructive reads at the beginning and end of the integration, which are then averaged to reduce the effective read noise.

The performances of the CAIN camera are summarised in Table 1.
Table 1. Main features of the CAIN camera relevant to this project.

\begin{tabular}{|c|c|}
\hline Detector & \\
\hline Gain (e $\mathrm{e}^{-} /$ADUs $)$ & 8.5 \\
\hline Well depth (at $2 \%$ linearity) (ADU) & -20000 \\
\hline Read-out noise ( $\mathrm{e}^{-}$, reset-read mode) & $40-50$ \\
\hline Read-out noise ( $\mathrm{e}^{-}$, Fowler mode, 4 reads) & $20-25$ \\
\hline Dark current $\left(\mathrm{e}^{-} / \mathrm{s}\right)$ & 5 \\
\hline Dead pixels & $<0.3 \%$ \\
\hline Sensitivity variation & $J<23 \%$ \\
\hline along the array (prior & $H<27 \%$ \\
\hline to flat-fielding) & $K_{\mathrm{s}}<25 \%$ \\
\hline \multicolumn{2}{|l|}{ Optics } \\
\hline Distortion $(1 \sigma$ of & $J: 0.01$ \\
\hline the averaged $F W H M$ over & $H: 0.08$ \\
\hline the array, in pix) & $K_{\mathrm{s}}: 0.03$ \\
\hline
\end{tabular}



Fig. 1. Transmission curves for the TCS-CAIN filters (dashed lines) and total system efficiency (solid lines), including the contribution from the filters and the detector (dotted line) and assuming an atmospheric absortion corresponding to a $4 \mathrm{~mm}$ water vapour column at the OT (upper solid line).

\section{Observation and data reduction}

In 1999 a group at the Instituto de Astrofísica de Canarias started a major observational programme to obtain deep NIR images in specific areas along the Galactic plane. The survey consists in 30 different observational campaigns, with an average of 5-6 nights per campaign with the CAIN camera at the 1.5-m Telescopio Carlos Sánchez (TCS) (Observatorio del Teide, Tenerife). The sky was surveyed in the $J, H$, and $K_{\mathrm{s}}$ bands (Fig. 1), in series of $20 \times 12 \operatorname{arcmin}^{2}$ fields, divided in a grid of $3 \times 5$ frames around the selected central position (although in some cases a $5 \times 5$ grid was used in the most interesting zones). CAIN has a FOV of $4.25 \times 4.25$ and the images were taken with an overlap of $20^{\prime \prime}$ between consecutive frames to give a total area in each field of approximately $0.07 \mathrm{deg}^{2}$. Figure 2 shows an example of a grid of observations covering a $20 \times 12 \mathrm{arcmin}^{2}$ area in 15 consecutive pointings.

\subsection{Survey strategy}

The survey positions are mainly distributed along or near to the Galactic plane $\left(|b| \leq 10^{\circ}\right.$, see Fig. 3), with the central position 


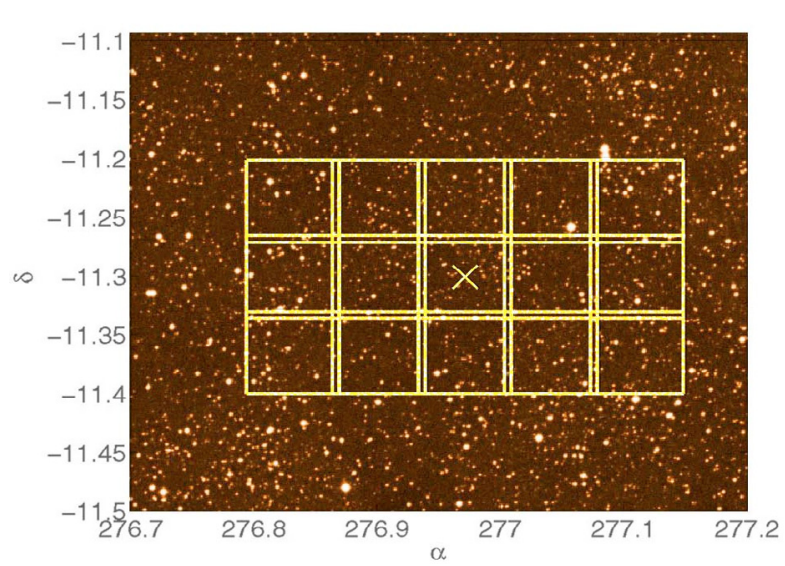

Fig. 2. Schematic view of an observed field that includes 15 consecutive pointings for a total of $20 \times 12 \operatorname{arcmin}^{2}$ coverage. The central mark corresponds to $l=20^{\circ} b=0^{\circ}$. The background image was taken from the ESO Online Digitized Sky Survey (http://archive.eso.org/dss/dss/).

of the field defined by its Galactic coordinates (and with the sky being scanned in strips of constant declination). For fields with $|b|>2^{\circ}$, there are only data corresponding to the $J$ and $H$-bands, as the Galactic extinction is almost negligible in the infrared this far from the plane, so the star counts in the $H$ and $K_{\mathrm{s}}$ filters are nearly equivalent. The total exposure times are $15 \mathrm{~s}$ both in the $J$ and $H(5 \times 3$ second exposures $)$ bands, and $16 \mathrm{~s}$ in the $K_{\mathrm{s}}$ band $(8 \times 2$ second exposures $)$. The observations were taken without guiding. On the $1.5 \mathrm{~m}$ TCS there is a sinusoidal tracking error in RA. This did not affect the individual images, but over the course of the $25 \mathrm{~s}$ needed to take the data in each filter, it meant that the source would typically move 2 to 3 pixels. This was useful as the source was measured by different pixels thereby helping to reduce the effect of bad pixels and giving a better sampling of the PSF. The images were taken using the "Fowler" readout mode. After the reset, the detector was read out five times with the first readout being discarded. There was then a pause for the integration time and a further five readouts were taken, again with the first of these being discarded. The sum from the first group of readouts and the second group of readouts were saved.

Nearly all data were collected under photometric conditions. Poor weather conditions lead to nearly $25 \%$ of the nights initially dedicated to the survey not being used, giving a total amount of 120 useful nights. Moreover, data obtained during clearly nonphotometric nights were repeated in a subsequent night with more favourable conditions (approximately 16 fields were repeated for this reason). In those cases, data finally collected in the catalogue are those that correspond to the night with the best seeing. Seeing conditions for those nights used varied from about 0.6 arcsec to over 2 arcsec. The seeing estimation is based on comparing the measured point spread functions with those predicted when seeing profiles are convolved with the 1 arcsec pixel scale. This 0.6 arcsec is close to the diffraction limit of a $1.5 \mathrm{~m}$ telescope. Figure 4 shows the seeing distribution for all the frames observed in the three filters of the survey, measured directly from the observations. The distribution is similar in the three cases, with mean values for the seeing of $\left\langle\sigma_{J}\right\rangle=$ $1.29 \operatorname{arcsec},\left\langle\sigma_{H}\right\rangle=1.22 \operatorname{arcsec}$, and $\left\langle\sigma_{K_{\mathrm{s}}}\right\rangle=1.24 \operatorname{arcsec}$ (for a total of $\sim 8300$ accepted frames $)$.

\subsection{Photometry and source extraction}

The data reduction was carried out with the $\operatorname{IRAF}^{1}$ and the DAOPHOT package (Stetson 1987). The final images in each filter are made up of 5 or 8 individual frames, with the sum of the initial and final readouts being saved separately. There were five readouts taken during the initial and final readouts. The first readout, however, was not used as with this particular detector there is a parasitic charge that builds up in the output of the detector when it is not being read out. To clear this charge, the detector is read out once immediately before the readouts are taken that are to be used. This means that the initial image has an effective integration time of $200 \mathrm{~ms}$ and the final image $200 \mathrm{~ms}$ + nominal integration time. The first step was to subtract a dark image taken with the same exposure time as used for the image. The resulting image was then examined for any pixels with more than $80 \%$ of the saturation level. In such cases these pixels were replaced with values from the initial readout multiplied by the factor (integration time $+200 \mathrm{~ms}$ )/ $200 \mathrm{~ms}$. In this way the dynamic range is increased by 2.5 to $3 \mathrm{mag}$, giving a saturation limit of about 6 to 7 rather than 9 to 10 . A number of tests were made using images with bright, but not saturated, sources to ensure that the fluxes given for the bright stars were correct and to find the correct flux level for usinge this method. Also, the linear range of the detector is large enough $(\sim 70 \%$ of full well) to ensure that there are no effects of non-linearity for the series of images like the ones used during the survey. The initial image was subtracted from the final image to give a frame with the nominal exposure time.

All of the frames in each filter in each area (i.e. $3 \times 5 \times 5$ or 8 frames) were then combined, using a median filter to remove the sources, to produce a sky image. This was then subtracted from each individual image to give a sky-subtracted image. The sky backgrounds measured in each $J$ band integration were typically $8 \%$ percent of the detector full well in the zenith, and in the $H$ band they were about twice this. The background varied linearly with airmass but not seasonally. In the $K$ band the background was typically $25 \%$ in summer and $15 \%$ in winter with less airmass dependence, and the majority of the flux comes from the telescope, not the sky. The cryostat window is very close to the focal plane, which means that any dust on the window is nearly in focus on the detector. Any flexure between the window and the detector as the telescope moves then causes this dust to create significant artifacts in the image at a level of up to $15 \%$ of the background flux. Although the flexure is under a pixel, it was sufficient to cause problems in the image analysis if the flat field was not obtained at the same position and the observation. Therefore it was decided to use the sky image as the flat field. Tests showed that the large-scale flat field was highly stable during an observing run.

The individual frames at each position were realigned, using the brightest sources detected in each frame, before combining them to produce the final image for that position. This also removed any cosmic ray events and other noise spikes.

DAOPHOT provides two measures of the reliability of a detection and of its photometric accuracy: the goodness of fit, $\chi^{2}$, and a measure of the image sharpness, sharp, indicating image blemishes and resolved objects. Detections with $\mid$ sharp $\mid>1$ have been eliminated, as this rejects the detections around highly saturated stars and extended sources. Another parameter that

1 IRAF is distributed by the National Optical Astronomy Observatories, which are operated by the Association of Universities for the Research in Astronomy, Inc., under cooperative agreement with the National Science Foundation. 


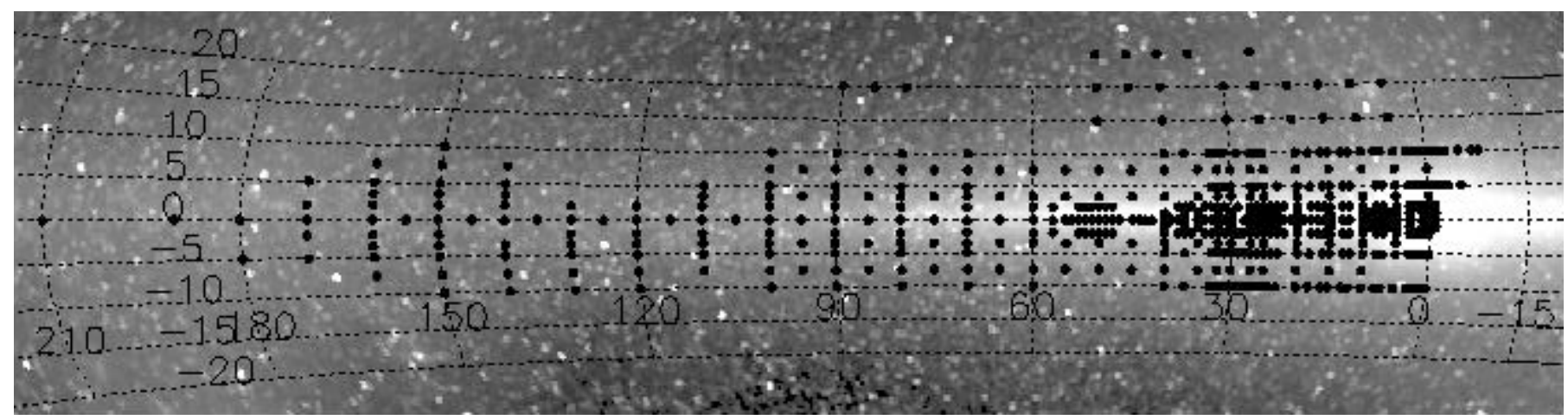

Fig. 3. Galactic fields observed along the TCS-CAIN survey super-imposed on a flux image of the Galaxy taken from the COBE/DIRBE (Boggess et al. 1992) web server (http://lambda.gsfc.nasa.gov/product/cobe/). As shown, pointings are mostly distributed in the inner Galaxy $\left(l<15^{\circ}\right)$ and around the near tip of the Galactic bar $\left(l=27^{\circ}\right.$, Hammersley et al. 2000; López-Corredoira et al. 2001). Each point corresponds to approximately $0.07 \mathrm{deg}^{2}$ in the sky (note they are not in scale).
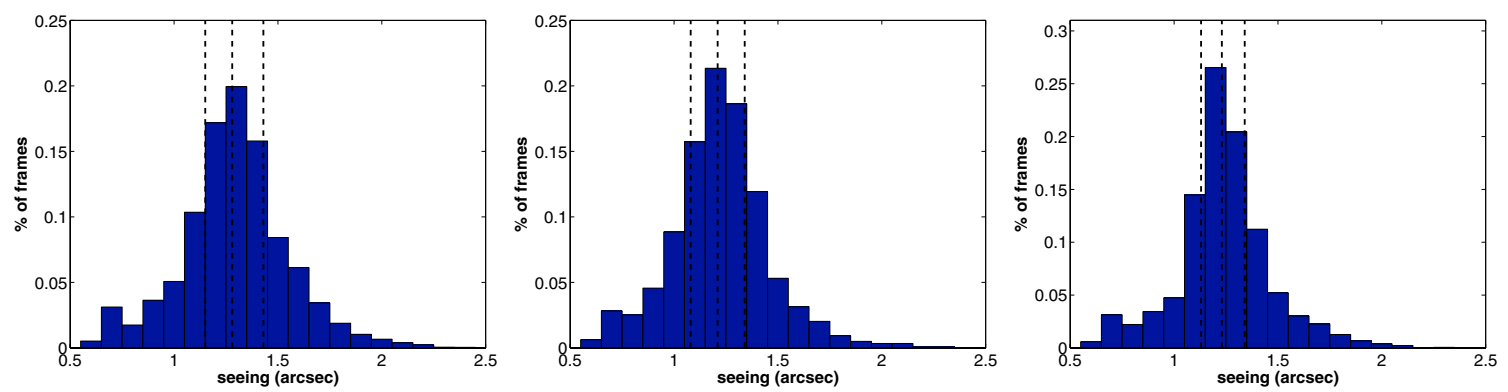

Fig. 4. Seeing distribution for all observed frames in $J$ (left), $H$ (centre), and $K_{\mathrm{s}}$ (right). Vertical dashed lines refer to 25 , 50 , and 75 percentiles of the distributions.
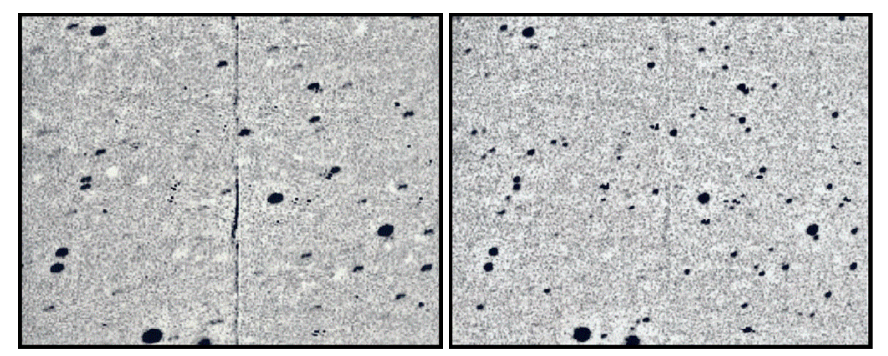

Fig. 5. Example of a poorly aligned image (left) that was detected by measuring the mean value of the round parameter in the image. The correctly aligned image is shown on the right.

DAOPHOT provides is round, which measures the ratio of the difference in the height of the best-fitting Gaussian function in $x$ minus the best-fitting Gaussian function in $y$, over the average of the best-fitting Gaussian functions in $x$ and $y$. This parameter allowed us to establish a preliminary estimate of the image reliability. Images with a mean round greater than 0.5 were marked and the reduction procedure was repeated, as this indicated that there was a problem with the realignment of the individual frames. Figure 5 shows an example where initially the realigned procedure did not work correctly (left image) and the process had to be repeated (right image), changing the parameters. Approximately 20 fields initially had this problem $(\sim 4 \%$ of the observed fields). As the round parameter is a reliable indicator of the quality of the final image, a flag for this has been included in the final version of the catalogue.

The final photometry was obtained using aperture photometry on the final image in each filter (Fig. 6 demonstrates the uniformity of the source extraction). In order to minimise confusion with neighbouring sources, the aperture size chosen was 2 arcsec. In order to correct for variations in the seeing, the seeing was determined for each image, which was then used to correct the instrumental magnitudes. This correction was calculated from a look-up table produced with artificial stars generated for different seeing conditions. Tests made with data from the same fields taken under different seeing conditions show a variability in the magnitudes of the order $0.001 \mathrm{mag}$ in the three filters, with a dispersion of $0.05 \mathrm{mag}$ in each band. Those values are coincident with the internal photometric accuracy of the survey (see Sect. 3.3), thus no effect due to the seeing condition must be expected in the photometry of TCS-CAIN.

The final $J H K_{\mathrm{s}}$ catalogue was made by cross-correlating the list of sources in each filter within a matching radius of $1.5 \operatorname{arcsec}$ of the $H$ band detections. The $H$-band was chosen as this has the most sources in common with the other two filters. Before the cross-correlation was done, a small magnification difference between $J, H$, and $K_{\mathrm{s}}$ had to be corrected. A final cross-correlation was then made with a smaller matching radius of 0.5 arcsec. In cases where there were two possible sources detected in one filter, which match a source in the second filter, the match for closest position was chosen. The detections in overlapped regions between adjacent frames are maintained until the astrometric reduction was done for further tests of the astrometric and photometric accuracy of the survey.

Data that have finally been reduced correspond to approximately 530 fields, with more than 10 million point sources detected simultaneously in the three filters, covering a total area of $\sim 41 \mathrm{deg}^{2}$ of the sky. 

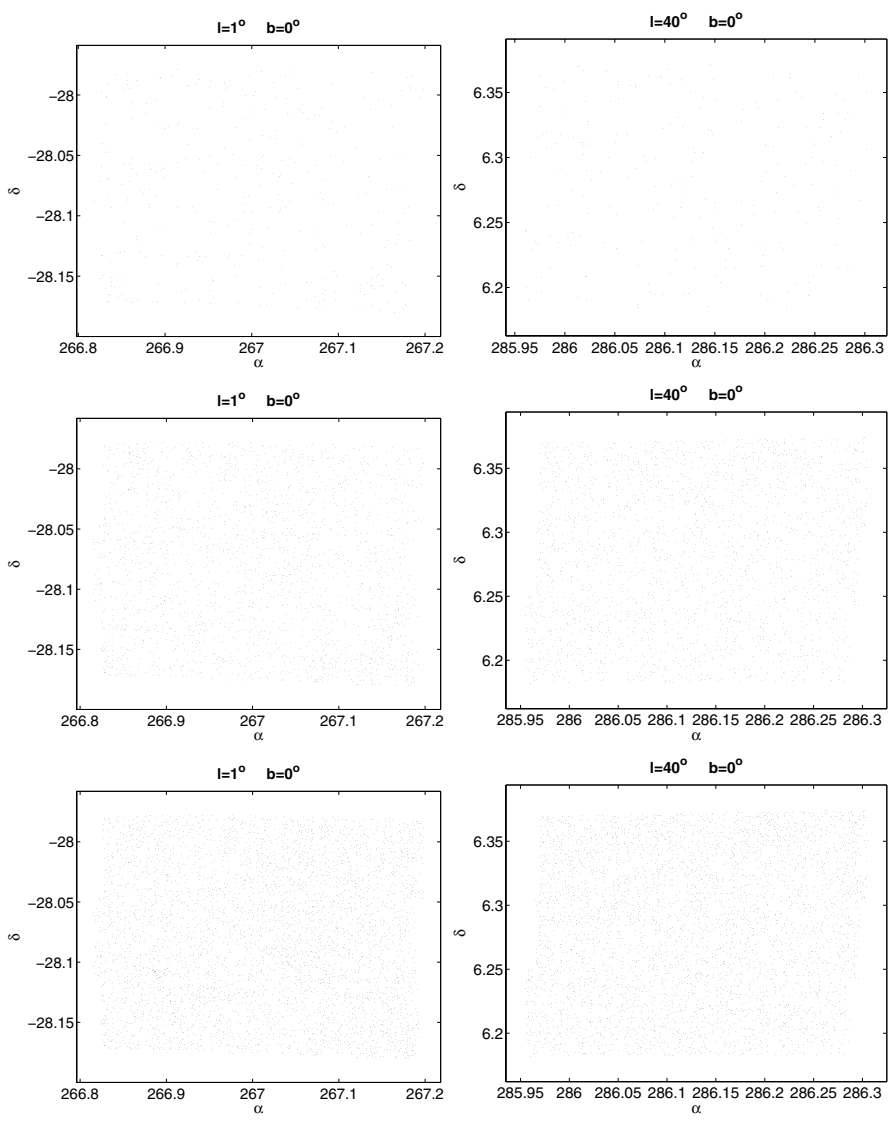

Fig. 6. Distribution of stars detected in the frames corresponding to the fields $l=1^{\circ} b=0^{\circ}$ (left) and $l=40^{\circ} b=0^{\circ}$ (right) at magnitudes brighter than $K_{\mathrm{s}}=10$ (upper panels), $K_{\mathrm{s}}=13$ (middle panels), and $K_{\mathrm{s}}=15$ (lower panels). Some structures can be shown due to the higher extinction in some regions of the fields.

\subsection{Photometric calibration}

Calibration was based on the faint star standards (FS) from the UKIRT catalogue (Hunt et al. 1998). These were observed each night at different airmasses to compute both the zero-point and the extinction coefficients for the night. No colour terms were considered because the effect of these terms is negligible in the infrared range. In fact, a comparison with 2 MASS photometry (see details in Sect. 3.5) yields a set of colour terms of the order of 0.02 mag each, thus in the more extreme cases we are dealing with corrections of $\sim 0.06 \mathrm{mag}$, well within the internal accuracy of the survey. Mean values obtained for the whole TCS-CAIN survey are summarised in Table 2 . When comparing with those estimated from the mean extinction curves of the Canarian observatories, we obtain slightly higher values than is expected for these wavelengths. This is in part due to some nights being effected by atmospheric dust (note that more than $40 \%$ of the observations were made in the summer when the dust is more common). Also there is some evidence of there being a small amount of flexure between the instrument and the pupil of the telescope, which gives up to $2 \%$ vignetting at very low airmasses when the system is aligned for the zenith. This vignetting appears to be highly reproduceable and so does not significantly effect the photometric accuracy of the survey. Figure 7 shows the the extinction coefficients obtained during the survey. The distributions are very similar and show how there is a lower dispersion as the wavelength increases, as was expected. The zero-points and
Table 2. Mean calibration coefficients for the TCS-CAIN survey.

\begin{tabular}{ccc}
\hline \hline Filter & Zero-point $(\mathrm{mag})$ & $k_{\lambda}\left(\mathrm{mag} \mathrm{airmass}^{-1}\right)$ \\
\hline$J$ & $21.159 \pm 0.028$ & $0.179 \pm 0.011$ \\
$H$ & $21.005 \pm 0.025$ & $0.148 \pm 0.012$ \\
$K_{\mathrm{S}}$ & $20.236 \pm 0.026$ & $0.167 \pm 0.013$ \\
\hline
\end{tabular}

extinction coefficients were calculated for each night, and each image was calibrated using the specific calibration for that night.

To estimate the internal precision in the photometry of TCS-CAIN, we used the overlapping regions between the frames where the same star will have two separate measurements. This was done for a control sample of 40 fields, which we consider a number statistically significant enough to be representative of the catalogue as a whole. In Fig. 8 we show the distribution in the difference in magnitude for the same star detected in separate images. As expected, well-defined and symmetric distributions around 0 are obtained, with mean values of $\langle\Delta J\rangle=-0.002 \mathrm{mag}$, $\langle\Delta H\rangle=-0.003 \mathrm{mag}$, and $\langle\Delta K\rangle=-0.001 \mathrm{mag}$. The dispersion of differences between the detected magnitudes is also similar $\left(\sigma_{J}=\sigma_{H}=0.16 \mathrm{mag}\right.$, and $\left.\sigma_{K}=0.13 \mathrm{mag}\right)$. However, it should be note that the edge of the CAIN detector has a number of defects, which significantly degrades the quality of the photometry in this region. Hence, the above are a lower limit for the reliability of the photometry, and the true values are approximately half this $(\sim 0.08 \mathrm{mag})$.

\subsection{Limiting magnitudes of TCS-CAIN}

The nominal limiting magnitudes of the TCS-CAIN survey are $17,16.5$, and 15.2 in $J, H$, and $K_{\mathrm{s}}$, respectively. These magnitudes were determined using growth curves of the differential star counts, taking the limiting magnitude to be where the curve departed by $5 \%$ from a linear extrapolation (Fig. 9). Differential star counts, however, are affected by the presence of Galactic components, such as the bulge or bar, which also make the growth curve go non-linear. Therefore, the range of magnitudes used for fitting was optimised for each field taking any expected component into account. This effect can be noted in Fig. 9, mainly in the $K$ and $H$ bands, where there is a flattening in the growth curves. For fields in the inner Galaxy $\left(l<30^{\circ}\right)$, the higher extinction, together with source confusion, produces brighter completeness-limiting magnitudes of 16.5 in $J, 15.4$ in $H$, and 14.6 in $K_{\mathrm{s}}$, or even less when close to the Galactic Centre. Figure 10 shows the variation in the completeness, field by field. Shown is the change in the limiting magnitude (defined by completeness in the counts) with Galactic longitude. This is equivalent to a representation of the dependence of the completeness with the source confusion, which is higher when closer to the Galactic Centre. The dashed lines show a moving average obtained using bins of $5^{\circ}$. As is shown, the completeness is almost constant (within the noise) for longitudes $l>35^{\circ}$, while for the inner Galaxy it decreases up to two magnitudes in each filter. Note that the moving average only shows a rough estimation of the completeness in each field, as there are other factors such as extinction or the location of structural components (as the bar and bulge) that affect individual fields.

The dependence of completeness on source density is an indicator of the confusion of the field. In Fig. 11 we show the variation in the completeness limit, as defined previously, with the number of 12 th mag stars per square degree in the $\mathrm{H}$ filter. As is shown in the central panel of Fig. 10, there are no fields 

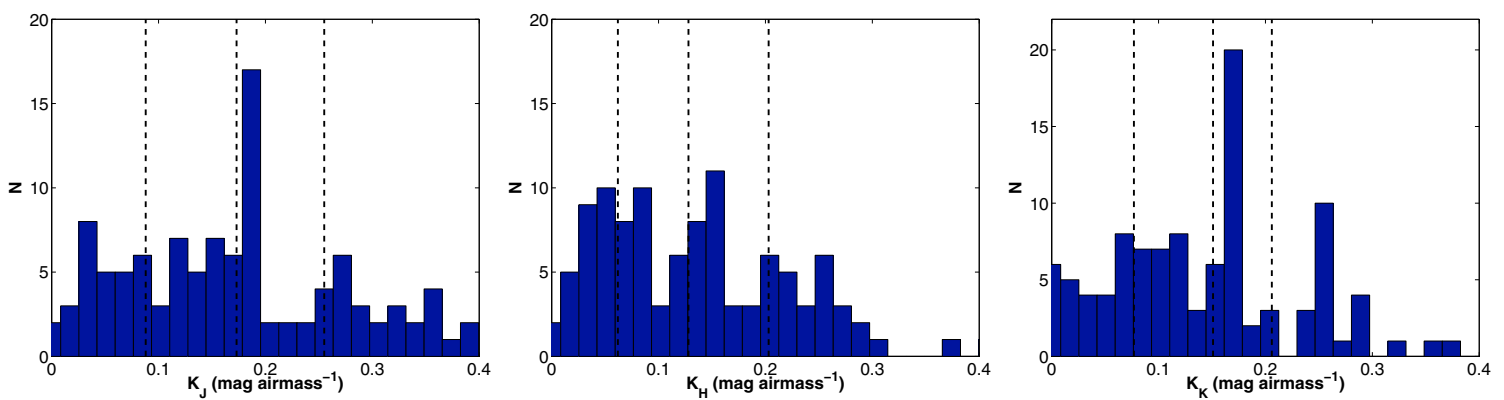

Fig. 7. Distributions of extinction coefficients in the three filters during the TCS-CAIN survey. As in Fig. 4, vertical dashed lines refer to 25 , 50, and 75 percentiles of the distributions.
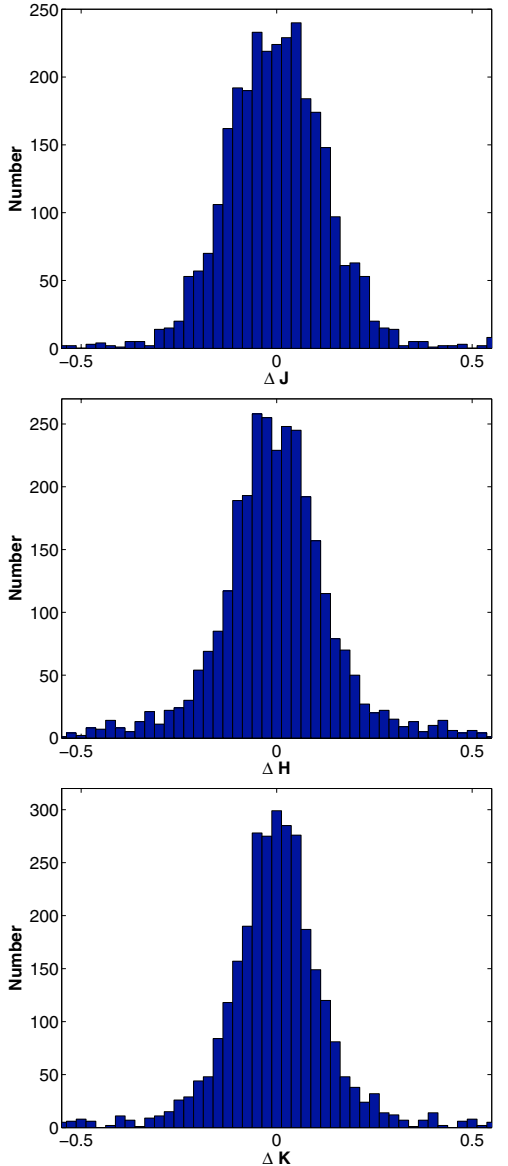

Fig. 8. Histograms of magnitude differences between objects lying in overlapped frames. Dispersion in the differences decreases as the filter becomes redder.

limited by confusion to magnitudes brighter than $m_{H}=12$. Again, for fields with a high source density, the completeness is about two magnitudes brighter than for the less crowded fields (see Fig. 11). The information about the specific completeness for each field will be given in the final version of the catalogue.

A series of observations were also performed where the total exposure times were increased by a factor 5 with the aim of obtaining deeper photometry in some specific areas of the sky. This gives data that are more than $1.2 \mathrm{mag}$ deeper than the nominal survey in the outer Galactic disc $\left(l>45^{\circ}\right)$, whereas in the inner Galactic disc there is no improved limiting magnitude, since the observations are limited by source confusion. An example of

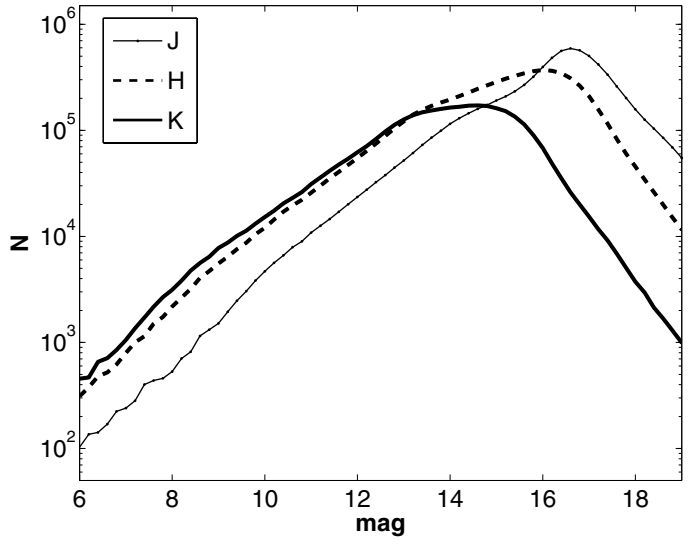

Fig. 9. TCS-CAIN cumulative star counts in bins of 0.2 mag for all the fields of the survey.

the increase in the completeness-limiting magnitude is shown in Fig. 12.

\subsection{Photometric comparison with 2 MASS}

The Two Micron All Sky Survey (2MASS, Skrutskie et al. 1997) is the largest NIR Galactic survey that is currently available. The TCS-CAIN survey cannot compete with 2MASS in terms of spatial coverage, as we obtain point source detection in regions of only $\sim 0.07 \mathrm{deg}^{2}$ in the sky. However, by using a smaller pixel size and larger integration times, TCS-CAIN can provide fainter photometry, since it is less affected by source confusion (see Fig. 13). This will be useful in analysing the inner Galaxy.

Once the astrometric reduction of the TCS-CAIN catalogue was complete (see a full description of this procedure in Sect. 4), we were able to compare the TCS-CAIN photometry with that of 2MASS using the cross identified sources (the 2MASS identifier for each source will be provided in the TCS-CAIN catalogue). The various flags provided in the 2MASS catalogue were taken into consideration when selecting sources for the astrometric reduction. The photometric differences also only used sources brighter than 15 in $J, 14$ in $H$ and 13 in $K$ s, well above the limiting magnitudes of both surveys (to avoid completeness effects in the differences). The distribution of differences for approximately $\sim 3 \times 10^{6}$ cross-matched stars are shown in Fig. 14 (this number corresponds to $\sim 30 \%$ of the total number of sources detected in TCS-CAIN). As it is shown, there is no significant shift in the magnitudes, with a distribution of differences with $\langle\Delta J\rangle=-0.015\left(\sigma_{J}=0.19\right),\langle\Delta H\rangle=0.013\left(\sigma_{H}=0.18\right)$, and $\left\langle\Delta K_{\mathrm{s}}\right\rangle=-0.011\left(\sigma_{K}=0.21\right)$. 

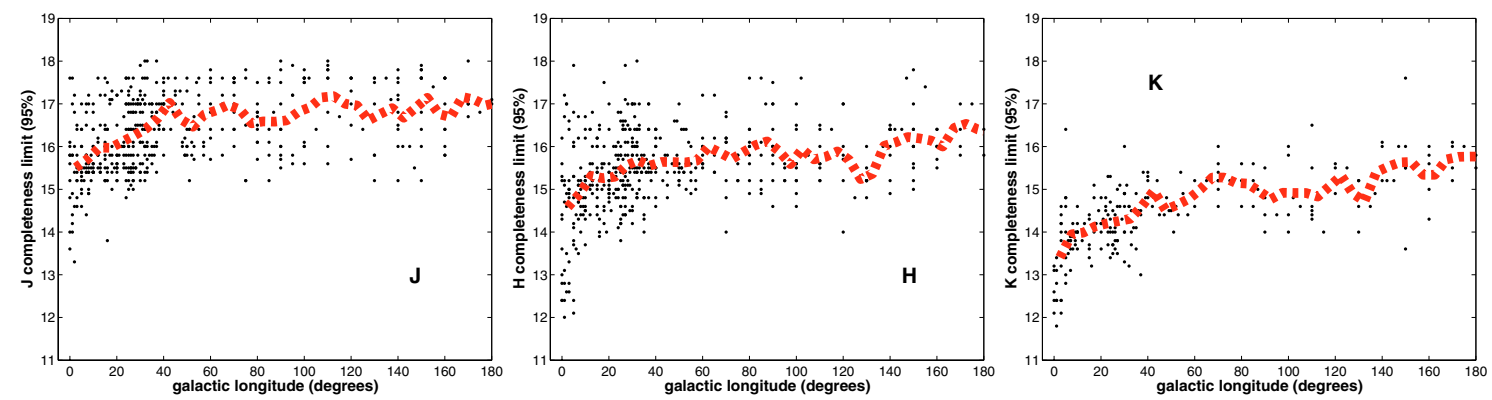

Fig. 10. Variation of the completeness-limiting magnitudes with the galactic longitude for the fields of the TCS-CAIN survey. Lines show a moving average obtained using bins of $5^{\circ}$.
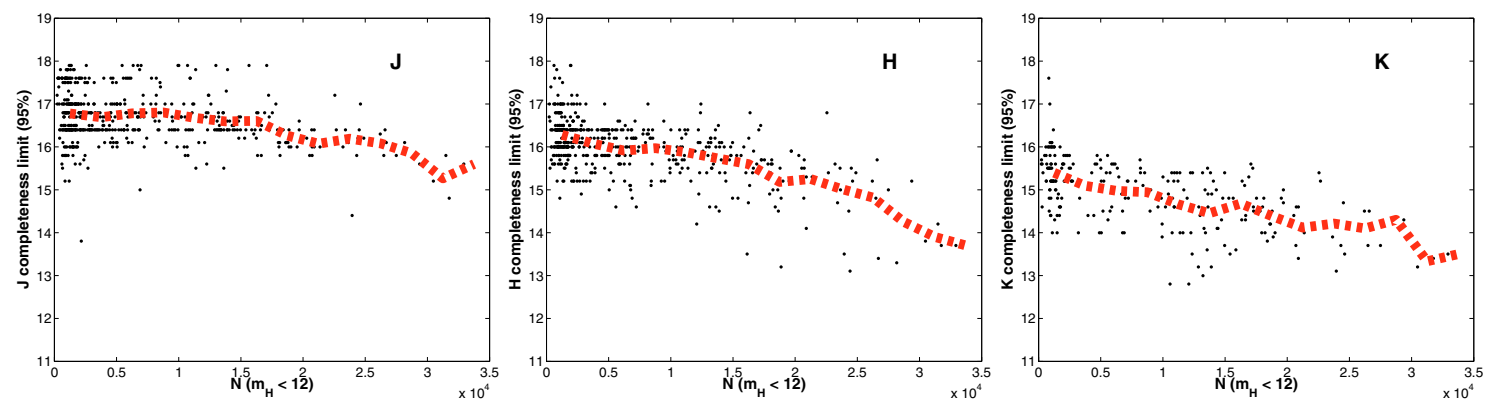

Fig. 11. Variation of the completeness-limiting magnitudes with the source density for the fields of the TCS-CAIN survey. Lines show a moving

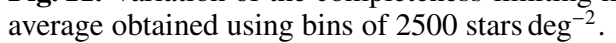

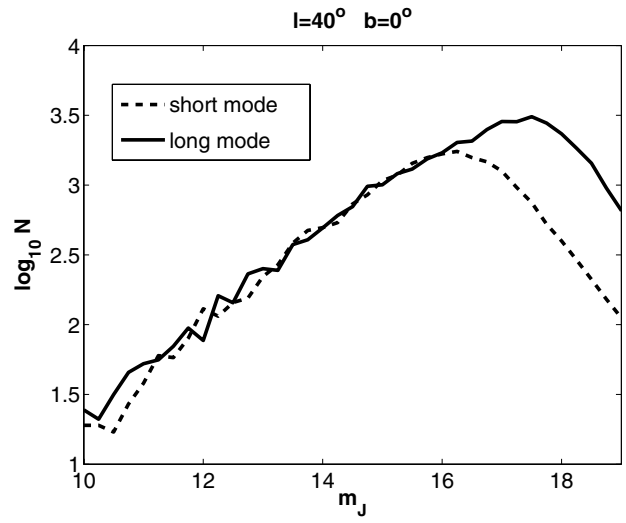

Fig. 12. Cumulative star counts in the $J$ band for the field $l=40^{\circ} b=0^{\circ}$ both in long and short observation modes. The observation is $1.5 \mathrm{mag}$ deeper when the long exposure is used (the differences that appear in the range $10<J<13$ are only due to a different binning of the data used).

We also computed colour transformations between 2MASS (2M) and TCS-CAIN (TCS) photometric systems obtaining a small dependence of the differences on 2MASS colours, as a result of not deriving colour terms during the TCS-CAIN calibration. However, for the redder stars we are dealing with corrections of $0.06 \mathrm{mag}$ (less than the internal accuracy of the survey), thus we can ignore their effect in the global photometry of TCS-CAIN.

$J_{\mathrm{TCS}}=J_{2 \mathrm{M}}-(0.015 \pm 0.006)(J-K)_{2 \mathrm{M}}+(0.033 \pm 0.005)$

$H_{\mathrm{TCS}}=H_{2 \mathrm{M}}-(0.024 \pm 0.008)(J-H)_{2 \mathrm{M}}+(0.062 \pm 0.002)$

$K_{\mathrm{TCS}}=K_{2 \mathrm{M}}-(0.025 \pm 0.003)(J-K)_{2 \mathrm{M}}+(0.071 \pm 0.008)$

$(J-K)_{\mathrm{TCS}}=(0.998 \pm 0.039)(J-K)_{2 \mathrm{M}}+(-0.006 \pm 0.002)$

$(J-H)_{\mathrm{TCS}}=(1.022 \pm 0.043)(J-H)_{2 \mathrm{M}}+(-0.002 \pm 0.001)$

$(H-K)_{\mathrm{TCS}}=(0.997 \pm 0.036)(H-K)_{2 \mathrm{M}}+(-0.001 \pm 0.001)$.

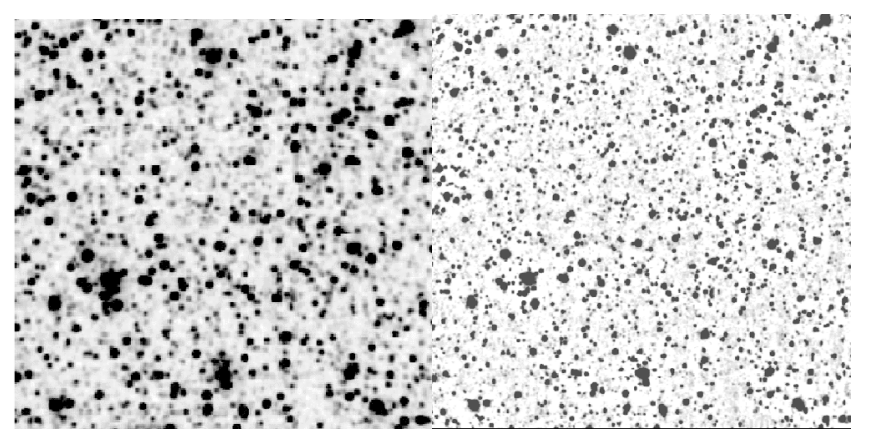

Fig. 13. $4^{\prime} \times 4^{\prime}$ images of $l=27^{\circ} b=0^{\circ}$ in the $K$-band taken from 2MASS (left) and TCS-CAIN surveys (right). As shown, the TCS-CAIN survey is notably less affected by source confusion.

A field-by-field analysis was done to show how variable the mean photometric differences between both surveys are. Figure 15 shows the distributions of mean differences for the Galactic fields observed in the survey. More than about $98 \%$ of the fields have differences lower than 0.4 mag in the three filters, while the percentage of fields with differences lower than $0.2 \mathrm{mag}$ is $87 \%$ (note that half of the fields have been not observed in the $K$ band), which is a reasonable estimate that supports the consideration of both surveys as photometrically equivalent. However, there are some fields with differences greater than $0.3 \mathrm{mag}$, which must be explained. As those fields are concentrated mainly in the run of July 2000, they most probably correspond to a bad photometric calibration due to a misidentification of the standard stars (there were several problems with the telescope pointing on those dates). Those fields with a significant offset compared to the 2MASS photometry (that is, greater than $0.2 \mathrm{mag}$ ) will be flagged in the final version of the catalogue.

As in Sect. 3.1 we also check the dependence of the differences with the source density of the fields. We again used the number of 12 th magnitude stars per square degree $\left(N_{\mathrm{H}}\right)$ as 

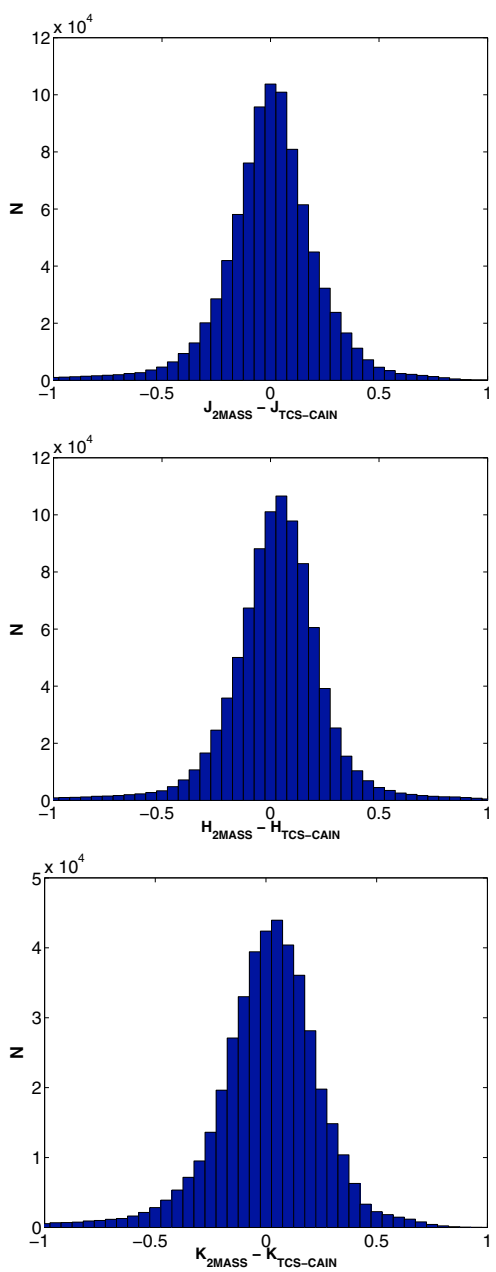

Fig. 14. Histograms of differences between TCS-CAIN and 2MASS magnitudes.

an estimate of the limit for source confusion, and obtained the photometric differences between 2MASS and TCS-CAIN, since this value changes from non-crowded fields (as do those of the anticentre) to highly crowded fields (as those of the innermost Galaxy). The results are shown in Fig. 16. As observed, there is no significant trend in the differences, showing a typical dispersion of values compared to the mean values obtained before in the whole range of densities. Thus, the values obtained for the mean differences are fairly stable and do not show any dependence on the crowding in the field.

This lack of dependence of the differences on the source density can be due to our photometric criteria of restricting the magnitude range of the cross-matched sources to values well above the completeness limiting magnitudes of both surveys. In order to check this issue, we computed the mean photometric differences between 2MASS and TCS-CAIN using different magnitude bins (see Fig. 17). As expected, the fainter that magnitude limit, the more negative the mean differences, as an effect of the higher source confusion present in 2MASS making the sources systematically brighter than in TCS-CAIN. As we mostly observed near Galactic plane fields in our survey, the effect of crowding must be relevant in the photometric comparison. As no differences were obtained in the range supposed to be "free" of confusion effects, we consider both surveys equivalent in this range, while we suggest that our magnitudes are more reliable than those of 2MASS when working with fainter magnitudes (that is, fainter than the limiting magnitudes of 2MASS: $J=15.8, H=15.1$, and $K=14.3$ ).

There is a slight trend in the difference in magnitude with magnitude. The slopes of a linear fit are $-0.004,-0.006$, and -0.008 for $J, H$, and $K_{\mathrm{s}}$, respectively, which implies that there is not a significant difference to $2 \mathrm{MASS}$. This effect is more noticeable as we approach the magnitude limit of 2MASS, suggesting that this is a possible origin. In the most extreme cases, differences are less than 0.05 mag between 2MASS and TCS-CAIN, consistent with both the distribution of differences of the whole sample of cross-matched stars (Fig. 14) and the internal accuracy of the TCS-CAIN survey $(\sim 0.08 \mathrm{mag})$.

A global photometric comparison of TCS-CAIN with DENIS is trickier due to the different sky coverage of this Southern Sky survey compared to TCS-CAIN. As obtained in Cabrera-Lavers \& Garzón (2003), photometric differences between 2MASS and DENIS filters $J$ and $K$ are negligible, so we can be confident in considering a similar relation between DENIS and our survey, since no differences have been found when comparing TCS-CAIN with 2MASS. In order to check that, we made a rough comparison in some test fields in common with the DENIS survey and obtained mean photometric differences of $\langle\Delta J\rangle=-0.01\left(\sigma_{J}=0.18\right)$ and $\left\langle\Delta K_{\mathrm{s}}\right\rangle=0.04$ $\left(\sigma_{K}=0.28\right)$. Those values agree well with our statement that 2MASS and DENIS are equivalent surveys in terms of photometry (Cabrera-Lavers \& Garzón 2003).

\section{Astrometric calibration}

An astrometric reduction is necessary to transform the instrumental coordinates of detected objects into celestial coordinates. Typically this involves describing the instrument's focal plane with a polynomial model, using an existing reference catalogue to determine the parameters of the model, i.e. the polynomial coefficients. A sufficient number of stars from the reference catalogue must be detected in each exposure to ensure that the model is well-determined. This model is then applied to all objects detected in the exposure, yielding celestial coordinates for all objects.

The astrometry in the TCS-CAIN survey was derived on a frame-by-frame basis, using a polynomial model to transform the $x, y$ positions into a standard reference frame. The reference star positions were taken from the 2MASS catalogue, which is itself on the system of the International Celestial Reference System (ICRS). The 2MASS catalogue was chosen for the reference as it has the high source density that is needed given the relatively small field of view of CAIN $(4.25 \times 4 ! 25)$, an ICRS precision of 100 mas, and because it is in the infrared it is substantially less plagued by extinction than the optical catalogues (e.g. USNO-B1.0, Monet et al. 2003).

The instrumental $x, y$ positions from the observations described in Sect. 3.2 were transformed into the 2MASS reference system by polynomial solution. As will be shown, a general third-order polynomial is adequate to describe the distortion caused by the telescope/camera combination. All detected sources were then transformed from the detector coordinates to sky coordinates to produce the final catalogue of J2000 coordinates.

\subsection{Astrometric pipeline}

The huge amount of data involved in the TCS-CAIN survey necessitates the use of a semi-automated pipeline for the astrometric reduction. This procedure works successfully for $95 \%$ of the 

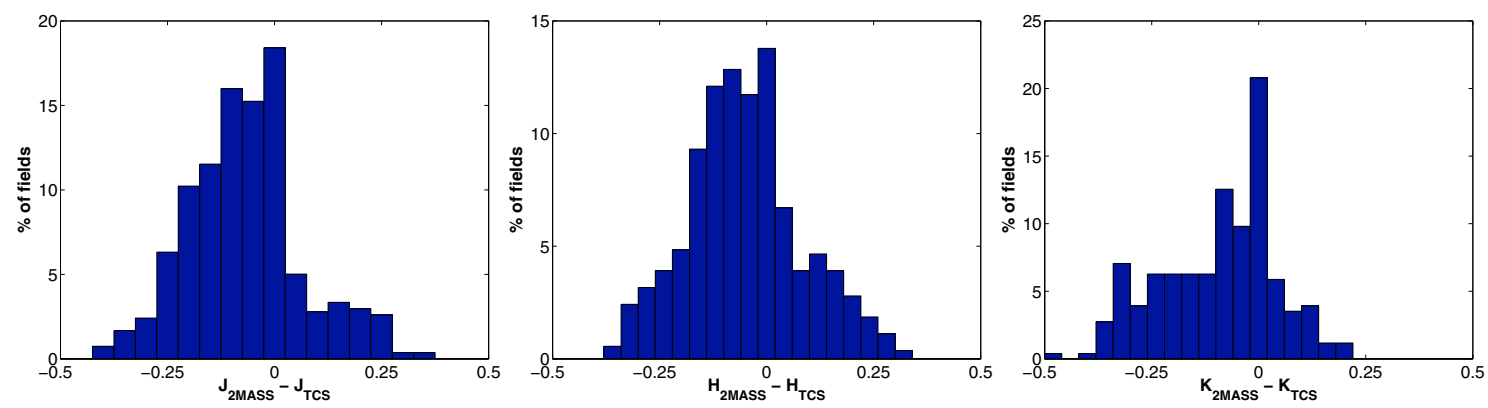

Fig. 15. Distributions of photometric differences in the three filters for the fields of the TCS-CAIN survey.
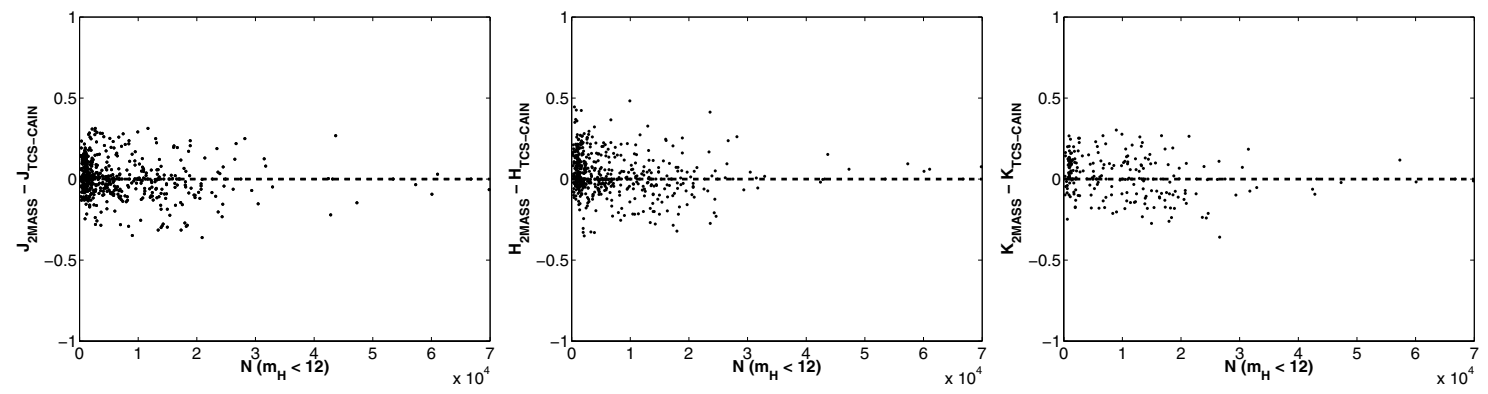

Fig. 16. Variation of the photometric differences in the three filters with the source density of the fields of TCS-CAIN surveys.

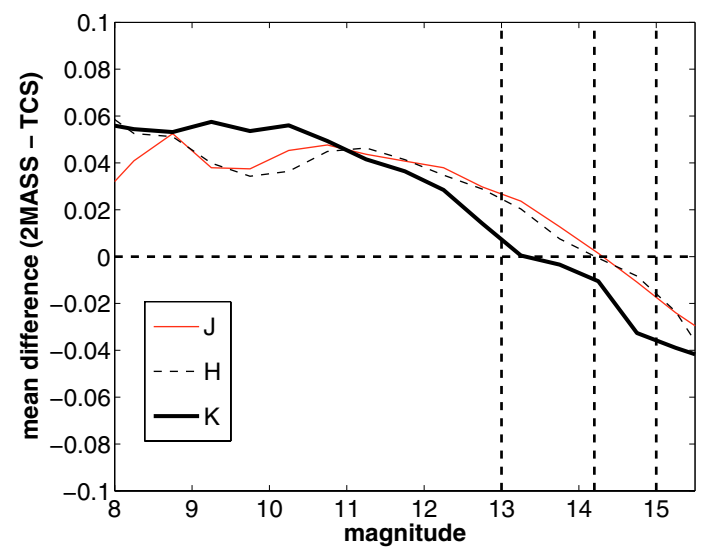

Fig. 17. Mean photometric differences between 2MASS and TCS-CAIN for different magnitude bins in the comparison. It is shown how, as the magnitude becomes fainter, the differences are more negative. Dashed vertical lines show the confusion-limited magnitudes of 2MASS for $K, H$, and $J$, respectively.

fields, with the remaining fields requiring some manual interaction. The frames with problems were primarily those where the number of available reference stars was low or those in which instrumental problems during the observations led to a long time delay in between the individual frames that made up an image.

The astrometry reduction was performed on each image. As there was only a small overlap between adjacent images (in fact, the overlap is null in some cases as shown in Fig. 18), there is little to be gained from more sophisticated overlap techniques. Thus, our procedure is to calculate an independent solution for each frame independently and then merge the results into a single catalogue.

The first step of the pipeline was to extract a rectangular region that is larger than the field being processed from the 2MASS catalogue. Although here 2MASS was used as the astrometric reference, the pipeline is ready to use any other catalogue
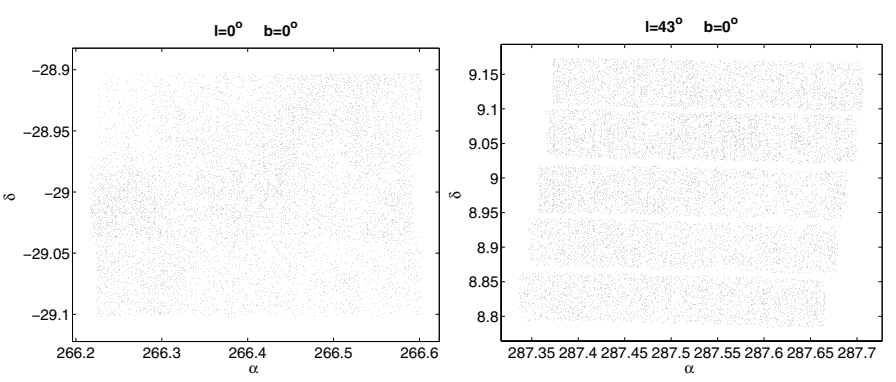

Fig. 18. Differences between two final astrometrically reduced images. The left panel shows a field with a correct overlapping, while the right panel shows a field where problems in the pointing of TCS eliminated the overlapping between the series of constant declination frames. This justifies finally discarding an astrometric reduction based on overlap techniques.

available in the future. Although the images were reduced individually, they were grouped by fields for practical consideration.

\subsection{Coordinate transformations}

The transformation from $(x, y)$ coordinates in an image into celestial coordinates $(\alpha, \delta)$ was determined using a general-purpose astrometric solution routine. This routine reads in the reference catalogue positions and, based on the nominal telescope pointing, makes a tangential projection of them onto the focal plane thereby producing standard coordinates $(\xi, \eta)$ for the reference stars. The goal is to find the polynomial transformation from measured $(x, y)$ coordinates to the reference stars' standard coordinates. The right ascension and declination axes are treated separately, i.e. as independent functions, and the polynomial coefficients are determined using a least-square fit.

The relevant coordinate transformations can be represented by the following symbolic scheme:

$(\alpha, \delta) \stackrel{\text { projection geometry }}{\longleftrightarrow}(\xi, \eta) \stackrel{\text { polynomials }}{\longleftrightarrow}(x, y)$. 
A star's celestial coordinates, $(\alpha, \delta)$, are projected onto the ideal focal plane as standard coordinates, $(\xi, \eta)$, via a simple geometric projection. The instrumental combination of telescope/instrument distorts these coordinates into the star's measured instrumental coordinates, $(x, y)$, and it is this second transformation that must be determined. To do so, a number of reference stars with known $(\alpha, \delta)$ must be identified in the frame. Their celestial coordinates are transformed to standard coordinates, and these are modeled as functions of the measured coordinates. The reference stars are used to determine the parameters of the model. The model is then applied to all measured stars, calculating their standard coordinates, which in turn are converted to celestial coordinates by inverting the geometric transformation.

\subsection{Polynomial solution for the transformation equations}

The routine used for identifying the reference stars is fully automatic and does not require cross-identification between the measured and reference catalogue stars. The program works by first making a preliminary cross-identification between the two input lists of stars, the detected stars, and the reference catalogue. This is done by searching for the brightest $\mathrm{N}$ stars in the two lists for a common pattern in the $(x, y)$ plane.

There was a choice of two possible algorithms to perform the pattern matching. The first is the triangle-matching algorithm of Valdés et al. (1995) implemented in the FOCAS software package. The second choice is a simpler difference-matching algorithm that uses a nominal pixel scale and orientation information to determine the vector offset that maximizes the correlation of the two lists in the standard coordinate plane, $(\xi, \eta)$. After some tests, it was decided to use the difference-matching algorithm as it was found to be more robust and less time-consuming than the triangle-matching algorithm.

Using this subset of cross-identified bright stars, a preliminary linear transformation was calculated and applied to all stars. This process generated a much more complete list of common stars, which could be used as reference stars in a new transformation solution; this time a cubic polynomial transformation was used. Following this, stars exceeding some cutoff value, typically residuals three times the standard error of the solution, were considered outliers and were eliminated in a way that did not introduce a bias. The process was repeated until no new matches were found and the solution became stable. The coefficients of the final solution were output.

Only those stars detected in both $J$ and $H$ filters were used as references. However, the transformation was applied to all detected sources, which were then saved. All of the above steps were handled by the pipeline without any manual intervention.

The routine also produces an improved estimate of the tangent point for the frame, based on the transformation solution. The recorded telescope position could be off by several arc minutes from the actual position $\left(\Delta \alpha_{0} \cos \left(\delta_{0}\right) \sim 29^{\prime \prime} \pm 75^{\prime \prime}, \Delta \delta_{0} \sim 39^{\prime \prime} \pm 37^{\prime \prime}\right)$. For this reason we chose first to find a linear solution that allowed the tangent point to be updated. A final solution was then made using a cubic polynomial function (as shown in Fig. 20).

The residuals of the reference stars (i.e. the difference between the position of the detected sources after being transformed and the catalogued coordinates of the reference sources) from all of the images can be co-added as a function of position in the focal plane and then plotted as a vector field. The stacked residuals from the first (linear) solutions will show any fixedpattern geometric distortions present in the telescope/instrument system. Such a plot from values obtained from interpolation can be shown as a field of vectors (left half of Fig. 20). The number of reference stars in each image is 290 stars on average (ranging from 30 to 890 depending on Galactic position), and 8313 images have been co-added. The resulting residuals diagram clearly reveals the systematic pattern. Almost all optical systems exhibit aberrations, among the most common being geometrical distortion. In this case the magnification depends on distance from the optical axis, i.e., position in the focal plane. Typically, the resulting radial displacement is proportional to $r^{3}$, although perfect azimuthal symmetry is not always seen. General cubic terms have been chosen in our final solutions to compensate for the distortion. The vector plot in the right panel half of Fig. 20, based on residuals from the final solutions, shows that no systematics errors remain in the positions, indicating that the cubic model is appropriate and has eliminated the fixed pattern distortion.

Following the astrometric reduction of the individual images in each field, they are merged into a single file. Stars in the overlap regions are matched by a positional tolerance of $1^{\prime \prime}$ and their multiple position and magnitude estimates are averaged. Note that no attempt was made to avoid statistical bias from the multiple opportunities for observing a faint source that appears in an overlapped area. This might produce some biases in modeling the star counts; however, the overlapped areas are very small ( $\sim 8 \%$ of the detector area) and there are several cases where the overlapping is null (as shown in Fig. 18), so we consider this effect negligible. Finally, the field sub-catalogues are combined to create the final TCS-CAIN catalogue.

\subsection{Distortion model for TCS + CAIN combination}

The coefficients of the polynomial model were determined independently for each image in the TCS-CAIN survey. By averaging the coefficients from the reductions of numerous images, a precise astrometric description of the TCS-CAIN focal plane and its distortions can be derived. We used Gaussian fitting to the distribution of model coefficient values for 8313 frames in order to arrive at a "best" description of the distortion of the TCS-CAIN focal plane.

The polynomial model used is

$$
\begin{aligned}
\xi= & a_{0}+a_{1} X+a_{2} Y+a_{3} X^{2}+a_{4} X Y+a_{5} Y^{2} \\
& +a_{6} X^{3}+a_{7} X^{2} Y+a_{8} X Y^{2}+a_{9} Y^{3} \\
\eta= & b_{0}+b_{1} X+b_{2} Y+b_{3} X^{2}+b_{4} X Y+b_{5} Y^{2} \\
& +b_{6} X^{3}+b_{7} X^{2} Y+b_{8} X Y^{2}+b_{9} Y^{3}
\end{aligned}
$$

where $X$ and $Y$ are the TCS-CAIN pixel coordinates, and $\xi$ and $\eta$ are the standard coordinates in arc seconds. This standard model includes scale and orientation (linear terms), correction to the tangential point (quadratic terms), and correction to the distortion (cubic terms).

The optimal values for the coefficients $\left(a_{i}, b_{i}\right)$ are summarised in Table 3 , while the constants $a_{0}$ and $b_{0}$ can be assumed to be zero, essentially reflecting the unknown telescope pointing error. Hence, if at least reference star can be identified in a frame, the position of the other sources can be determined.

The cubic terms represent the distortion of the telescopecamera. "Classical" distortion can be expressed as follows,

$$
\Delta x=R x\left(x^{2}+y^{2}\right) \quad \Delta y=R y\left(x^{2}+y^{2}\right)
$$

where $R$ is the distortion coefficient. Thus, it is seen that the TCS-CAIN system exhibits classical distortion, with 

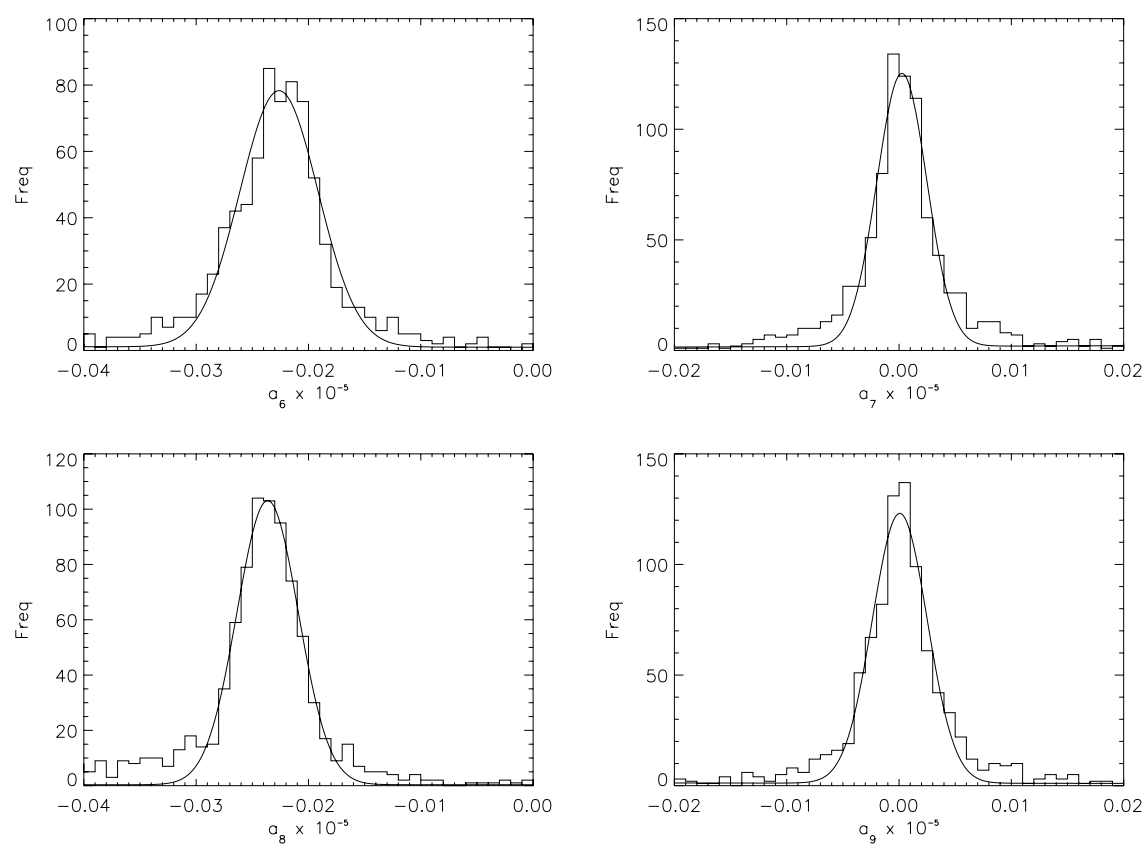

Fig. 19. Distribution of the derived values of the cubic coefficients for the $\alpha$ solution. The plots for the $\delta$ solution are similar. These plots and equivalent ones for the linear and quadratic coefficients were used to determine the "optimal" values.

Table 3. Optimal coefficients for TCS+CAIN combination.

\begin{tabular}{cc}
\hline \hline$a_{1}=1.00792$ & $b_{1}=0.00925$ \\
$a_{2}=-0.00782$ & $b_{2}=1.00156$ \\
$a_{3}=0.29929 \times 10^{-5}$ & $b_{3}=0.07308 \times 10^{-5}$ \\
$a_{4}=-0.03359 \times 10^{-5}$ & $b_{4}=-0.14984 \times 10^{-5}$ \\
$a_{5}=0.12857 \times 10^{-5}$ & $b_{5}=0.89969 \times 10^{-5}$ \\
$a_{6}=-0.02263 \times 10^{-5}$ & $b_{6}=-0.02252 \times 10^{-5}$ \\
$a_{7}=-0.00026 \times 10^{-5}$ & $b_{7}=-0.00044 \times 10^{-5}$ \\
$a_{8}=-0.02364 \times 10^{-5}$ & $b_{8}=-0.02258 \times 10^{-5}$ \\
$a_{9}=0.00006 \times 10^{-5}$ & $b_{9}=0.00007 \times 10^{-5}$ \\
\hline
\end{tabular}

$a_{7} \approx a_{9} \approx b_{7} \approx b_{9} \approx 0$ and $a_{6} \approx a_{8} \approx b_{6} \approx b_{8} \approx$ $0.02284 \times 10^{-5} \operatorname{arcsec~pixel}^{-3}$.

We suggest the above distortion model and coefficients be adopted for the TCS-CAIN instrument system. The values of the coefficients of the distortion model listed above, in particular the higher-order terms, are averages over thousands of frames and more than one hundred nights. As such, they are welldetermined, much more so than could be derived from a single exposure. Note the well-behaved, narrow distributions of the cubic coefficients plotted in Fig. 19.

\subsection{Estimation of errors}

The astrometric uncertainty in the TCS-CAIN catalogue can be estimated in two ways. Using the limited number of objects that lie in the narrow overlap region of adjacent frames, an internal error estimate can be made. Also, comparison can be made to an external catalogue of high precision, as discussed in the next section.

The distributions of positional differences based on the sources measured twice because they were found in the overlap region are shown in Fig. 21. The distributions show a Gaussian core with somewhat extended wings, probably a result of poorly fit images at the extreme border of the detector. Thus, errors
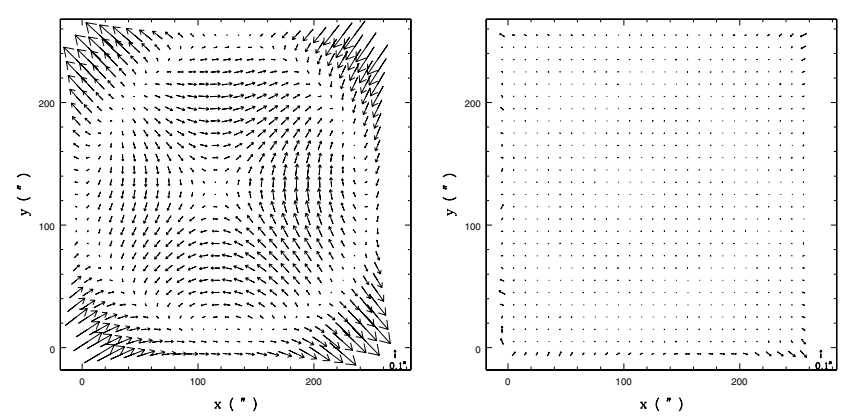

Fig. 20. Stacked position residuals as a function of the chip coordinates $x$ and $y$ after the first step in the reduction, when only a linear solution is calculated and applied. The fixed pattern shows cubic distortion (left panel). Distortion was corrected effectively when we used cubic terms in the final solution (right panel).

obtained in that way must be considered as a pessimistic estimate. Probability plot analysis indicates that the dispersion of the differences is $\sigma_{\alpha \cos \delta}=0 \prime^{\prime} 14$ and $\sigma_{\delta}=0 . ' 19$. Therefore the single-measurement internal error estimate is $\sigma_{\alpha \cos \delta}=0.10$ and $\sigma_{\delta}=0 . ' 13$.

Since the 2MASS catalogue was used as our astrometric reference catalogue, it is not surprising that when using it as an "external" comparison, the mean differences with the TCS-CAIN positions are zero. The standard deviation for these differences is $\left(\sigma_{\alpha \cos \delta}, \sigma_{\delta}\right)=\left(0{ }^{\prime} 16,0{ }^{\prime} 19\right)$. As expected, the TCS-CAIN catalogue is on the system of 2MASS, as illustrated in Fig. 22, to within a systematic uncertainty of 0 ' $^{\prime} 05$. Also, position difference vs. colour was investigated and no systematic difference was found (Fig. 23).

A more intertesting choice for an external comparison is the UCAC2 catalogue (The Second US Naval Observatory CCD Astrograph Catalogue, Zacharias et al. 2004). The UCAC2 is a high density, highly accurate astrometric catalogue of 48330571 stars covering the sky from -90 to +40 degrees in 

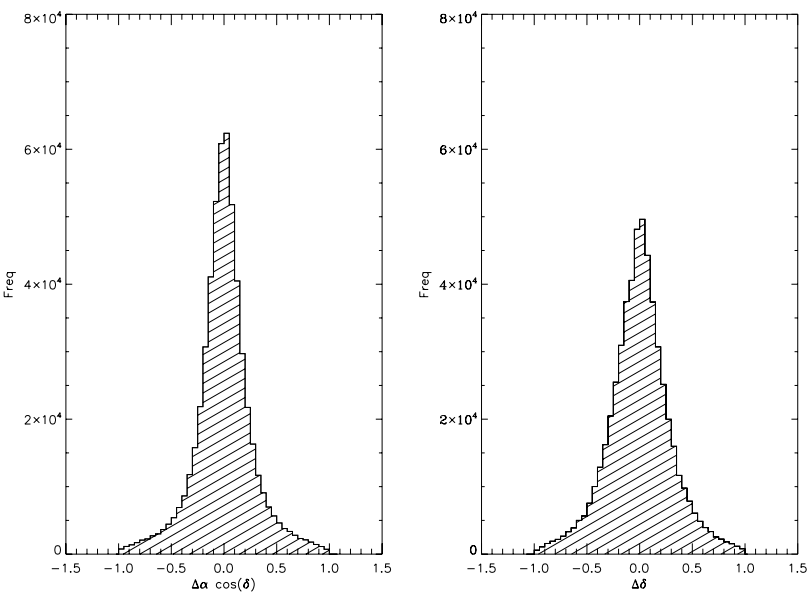

Fig. 21. Histograms of the internal errors obtained as differences between overlapped images (a total of $\sim 600000$ stars have been used for these plots). We obtain a very narrow distribution of differences that shows a Gaussian core in both cases.
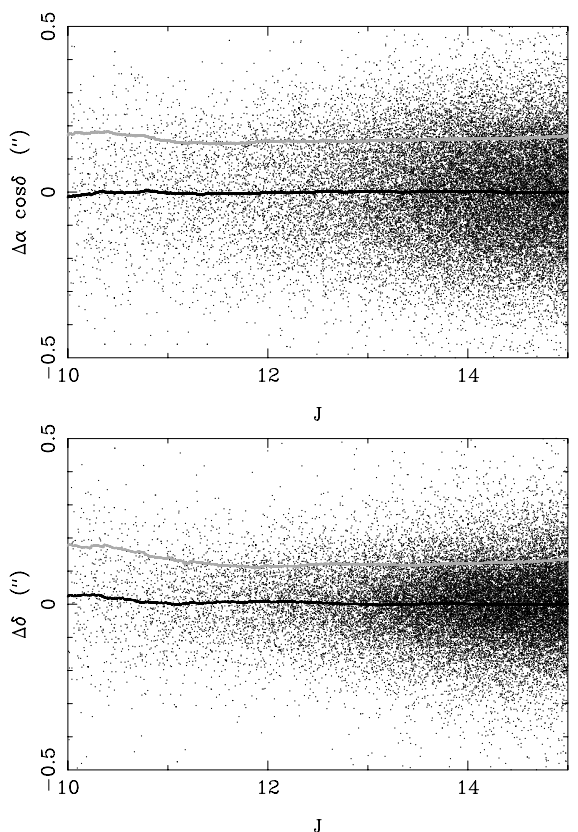

Fig. 22. Differences in positions between TCS-CAIN and 2MASS in right ascension and declination as a function of $J$ magnitude, for stars in common (we represent a sample of $\sim 30000$ stars for simplicity). For $H$ and $K_{\mathrm{s}}$ magnitudes we get similar plots. The dark line is the moving average and the grey line indicates the standard deviation.

declination. Proper motion and photometry are provided for all stars, with positions and proper motions being on the ICRS and given at the epoch J2000.0. Its estimated positional precision is about $0 .{ }^{\prime} 020$ for the stars in the 10-14 mag range and about to 0 '.070 $^{\prime}$ at the limiting magnitude of $R \sim 16$. These errors are negligible relative to the TCS-CAIN uncertainties, and thus the dispersion of the positional differences will reflect the errors in TCS-CAIN as the errors in the two catalogues will add in quadrature. We compared 12 test fields with the UCAC2 catalogue. Fields were selected to cover different galactic coordinates, thus covering different conditions for the source density. The distributions of positional differences between TCS-CAIN and UCAC2 are shown in Fig. 24 as a function of magnitude, for a total of $\sim 2500$ stars cross-matched between both catalogues.
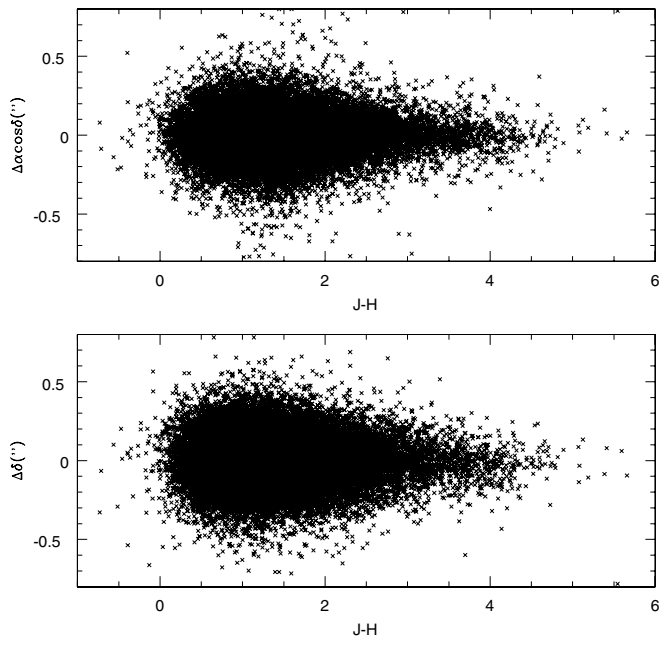

Fig. 23. Differences in positions between TCS-CAIN and 2MASS in right ascension and declination as a function of $J-H$ colour. No systematic dependence in the differences with the colour is observed (we have represented the same sample of stars as in Fig. 22).
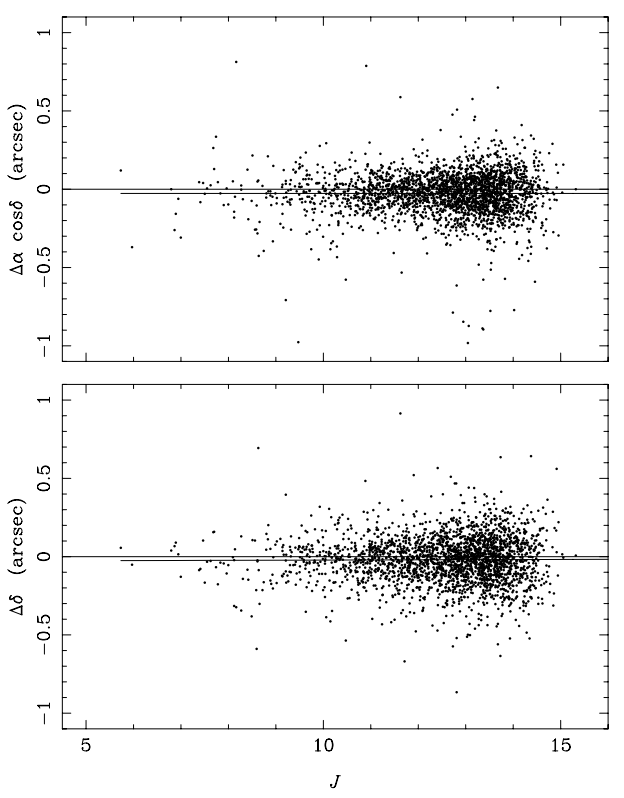

Fig. 24. Differences in positions between TCS-CAIN and UCAC2 in right ascension and declination as a function of $J$ magnitude. The horizontal line is actually a least-square linear fit to the differences, indicating that no magnitude equation is present.

The mean differences in $(\alpha \cos \delta, \delta)$ are $\left(-0{ }^{\prime} 03,-0{ }^{\prime} 02\right)$, and the dispersions of the differences are $\left(00^{\prime} 14,00^{\prime} 15\right)$. The dispersions are undoubtedly dominated by the TCS-CAIN positional errors, so these values are a reliable estimate of the TCS-CAIN positional uncertainties. It is important to note the lack of any trend with the magnitude of the sources in the TCS-CAIN/UCAC2 differences (Fig. 24). Assuming the positional accuracy of the UCAC2 does not have a magnitude dependence, which indicates that the TCS-CAIN positions are also free of any obvious "magnitude equation" (the undesired correlation between the position of the image centre and the magnitude of the star producing the image). 

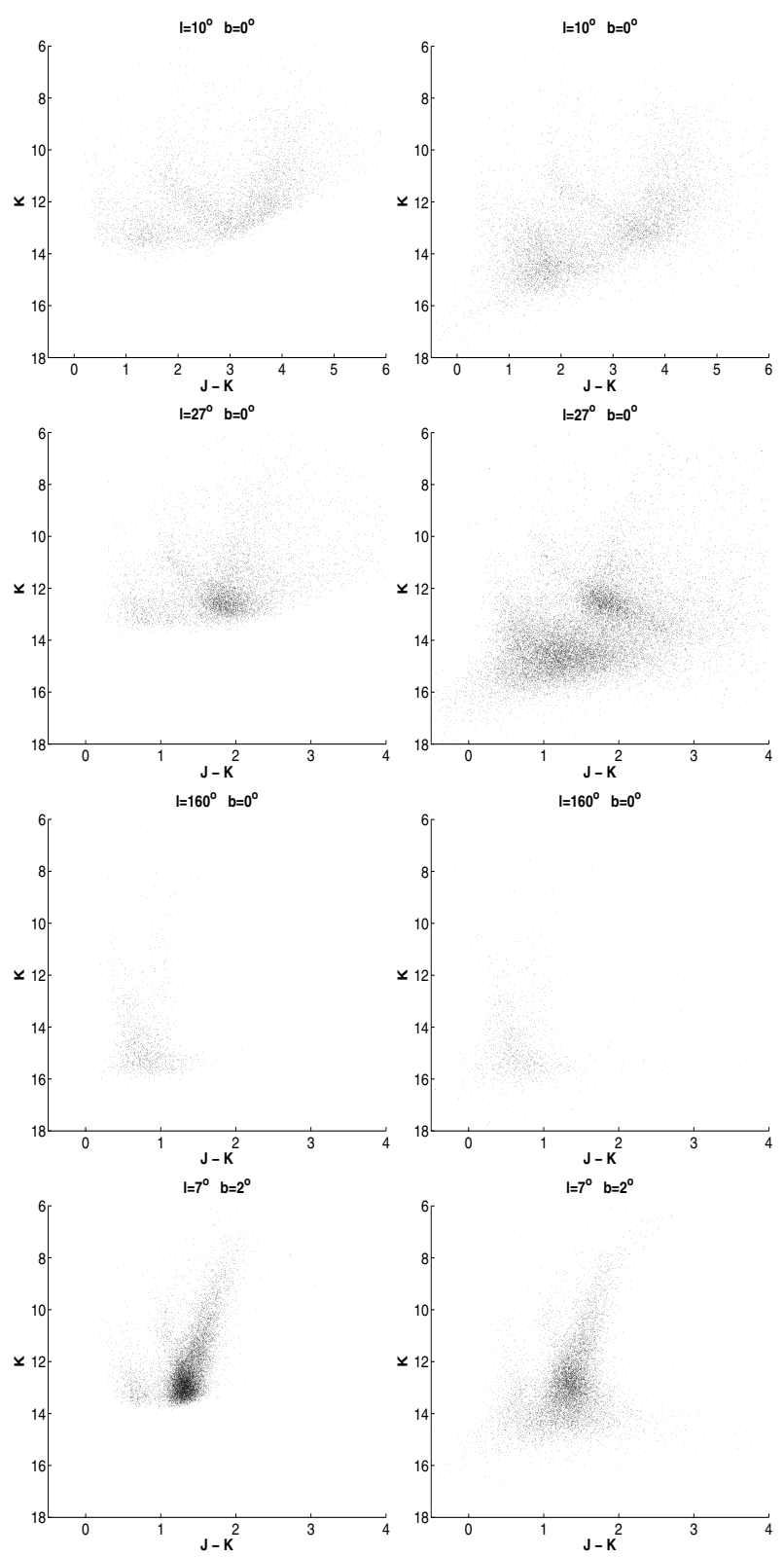

Fig. 25. Comparison between $K / J-K$ colour-magnitude diagrams obtained with 2MASS data left panels) and TCS-CAIN (right panels). As shown, the TCS-CAIN survey is clearly deeper than 2MASS in the $K$ band for $l<90^{\circ}$ (about $2 \mathrm{mag}$ in $\mathrm{K}$ for $l=27^{\circ} b=0^{\circ}$ ), without noticeable differences for $l>90^{\circ}$. This provides a better analysis of the Galactic structure of the inner Milky Way.

\section{TCS-CAIN NIR star counts. Implications in Galactic structure}

The TCS-CAIN data were used in various analyses of the largescale Galactic structure. A major cluster of stars was found on the Galactic plane at a distance of about $6 \mathrm{kpc}$ at $l=27^{\circ}$, which extends towards the Galactic Centre. This was shown to be the near tip of the in-plane Galactic bar (Hammersley et al. 2000). Analysing the position of the red clump giants in NIR colour-magnitude diagrams (CMDs) obtained with TCS-CAIN data provides an empirical method for obtaining both the interstellar extinction and the stellar density along a selected line of sight (Picaud et al. 2003; Drimmel et al. 2003; López-Corredoira et al. 2002, 2004; Cabrera-Lavers et al. 2005). More recently, Babusiaux \& Gilmore (2005) and Nishiyama et al. (2005) have
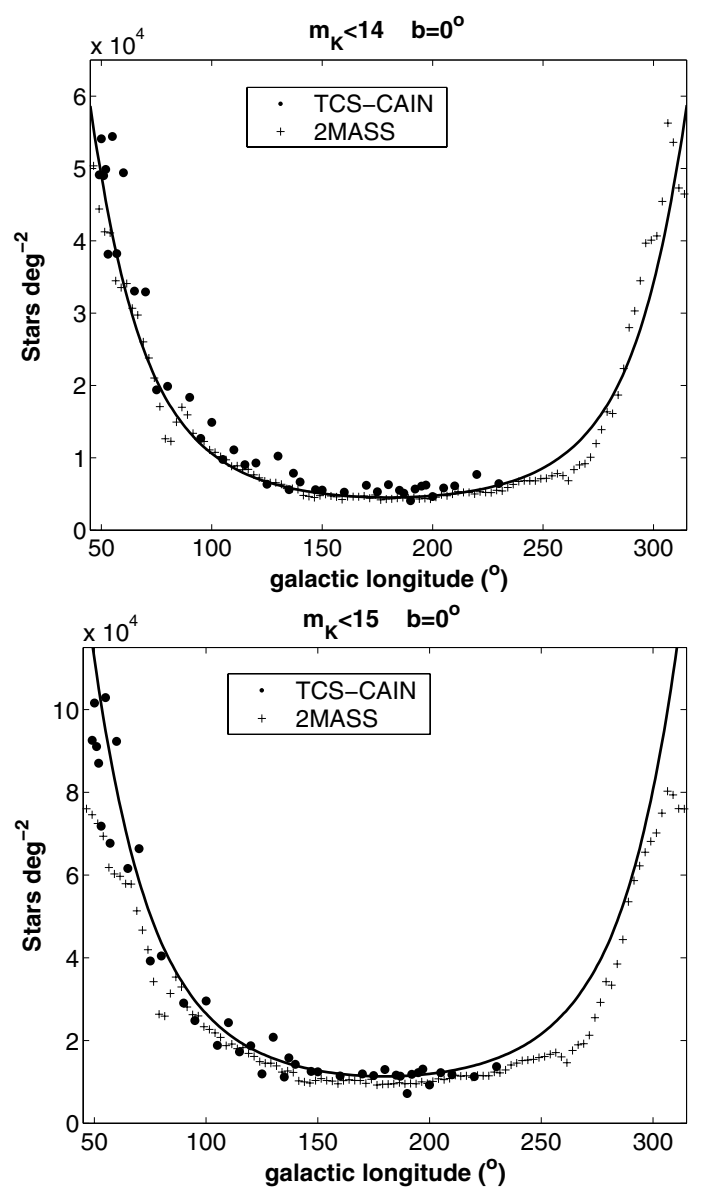

Fig. 26. Star counts in the plane by combining data from 2MASS and TCS-CAIN. The solid line is the prediction of the L02 model. We observe that TCS-CAIN counts fit well within the predictions of the model and that the 2MASS counts up to $m_{K}<15$ lie below the model, due to the effects of completeness.

analysed the structure of the inner Galaxy by using the red clump giants as a distance indicator. For those analyses they used propriety-deep NIR data, because 2MASS is too shallow to reach the red clump star in the CMDs in the inner Galaxy. The TCS-CAIN data set is deep enough to trace the red clump stars into the bulge (for a comparison between CMDs obtained with 2MASS and TCS-CAIN see Fig. 25), and although the coverage is not complete, the sampling is regular along the plane.

\subsection{Comparison with a Galactic disc model}

López-Corredoira et al. (2002, hereafter L02) present a model of the outer parts of the stellar Galactic disc based on the 2MASS point-source survey. This model suggests a density that falls exponentially with the Galacto-centric radius, $R$, and with the height $z$ (variable scale height $h_{z}(R)$ ). The scale height is variable due to the flare, which distributes the stars further from the plane with increasing Galacto-centric distance. The model also includes the effect of the warp, which produces asymmetries in the star counts. In order to estimate the accuracy in the combination of 2MASS and TCS-CAIN star counts, we compared the predictions of this model, whose parameters were obtained only by using 2MASS stars counts, with the combination of data from 2MASS and TCS-CAIN. Figure 26 shows the combination of star counts both from 2MASS and TCS-CAIN and the predictions of the L02 model for the Galactic plane for two different 


\begin{tabular}{|c|c|c|c|c|c|c|c|c|c|c|c|}
\hline id & RA_J2000 & DEC_J2000 & 1 & $b$ & $x$ & $y$ & $\mathrm{~J}$ & $\mathrm{H}$ & K & ing & 2MASS \\
\hline $\operatorname{cain} 102+020-110001$ & $330 . \overline{9} 91596$ & $57 . \overline{7} 45565$ & 102.063349 & 1.828306 & 47.31 & 2.19 & 99.999 & 15.128 & 14.814 & 2 & $22035794+5744436$ \\
\hline cain102+020-110002 & 331.003010 & 57.745797 & 102.068389 & 1. 824873 & 68.79 & 2.37 & 99.999 & 18.611 & 99.999 & 1 & \\
\hline cain102+020-110003 & 331.047538 & 57.745731 & 102.087479 & 1. 810707 & 153.41 & 2.38 & 14.045 & 13.579 & 12.998 & 2 & $22041138+5744446$ \\
\hline cain102+020-110004 & 331.097633 & 57. 746291 & 102.109342 & 1. 795295 & 249.73 & 2.25 & 99.999 & 17.901 & 99.999 & 1 & \\
\hline cain102+020-110005 & 331.058827 & 57.746134 & 102.092569 & 1. 807455 & 174.98 & 2.88 & 99.999 & 18. 213 & 99.999 & 1 & \\
\hline cain102+020-110006 & 331. 019221 & 57.746110 & 102.075538 & 1. 819985 & 99.69 & 3.43 & 16.916 & 16.844 & 99.999 & 1 & $22040461+5744457$ \\
\hline cain102+020-110007 & 331.072450 & 57.746364 & 102.098560 & 1. 803326 & 200.85 & 4.07 & 16.139 & 16.633 & 15.475 & 2 & $22041737+5744467$ \\
\hline cain102+020-110008 & 331.074361 & 57.746648 & 102.099550 & 1. 802949 & 204.70 & 4. 34 & 99.999 & 17.270 & 99.999 & 1 & \\
\hline cain102+020-110009 & 331. 095961 & 57.746648 & 102.108835 & 1. 796112 & 245.85 & 4. 40 & 15.083 & 15.181 & 14.531 & 2 & $22042306+5744477$ \\
\hline cain102+020-110010 & 330.988061 & 57.746412 & 102.062335 & 1. 830108 & 40.16 & 4.58 & 99.999 & 17.830 & 99.999 & 1 & \\
\hline cain102+020-110011 & 330.996901 & 57.746626 & 102.066258 & 1.827477 & 57.14 & 5.39 & 99.999 & 17.733 & 99.999 & 1 & \\
\hline cain102+020-110012 & 331.014957 & 57.746412 & 102.073886 & 1. 821580 & 91.82 & 5.29 & 15.292 & 15.059 & 14. 703 & 2 & $22040360+5744470$ \\
\hline cain102+020-110013 & 331.039783 & 57. 746691 & 102.084717 & 1.813936 & 138.76 & 5.29 & 99.999 & 17.224 & 99.999 & 1 & \\
\hline cain102+020-110014 & 331.071103 & 57.746883 & 102.098289 & 1. 804170 & 198.45 & 5.29 & 99.999 & 17.202 & 99.999 & 1 & \\
\hline cain102+020-110015 & 331.033794 & 57.746754 & 102.082181 & 1. 815885 & 127.39 & 5.61 & 16.866 & 16.339 & 99.999 & 1 & $22040811+5744479$ \\
\hline cain102+020-110016 & 330.969843 & 57.747000 & 102.054863 & 1. 836360 & 4. 30 & 6.14 & 17.832 & 19.023 & 99.999 & 3 & \\
\hline cain102+020-110017 & 330.984108 & 57.746588 & 102.060742 & 1. 831503 & 32.62 & 6.02 & 16.900 & 16.694 & 15.512 & 3 & $22035605+5744477$ \\
\hline cain102+020-110018 & 331. 000401 & 57.746873 & 102.067908 & 1. 826565 & 63.85 & 6.29 & 99.999 & 17.001 & 99.999 & 1 & \\
\hline cain102+020-110019 & 331. 022280 & 57.746920 & 102.077334 & 1. 819667 & 105.53 & 6.35 & 99.999 & 17.703 & 99.999 & 1 & \\
\hline cain102+020-110020 & 331.036278 & 57. 746921 & 102.083348 & 1. 815232 & 132.11 & 6.18 & 99.999 & 17.826 & 99.999 & 1 & \\
\hline
\end{tabular}

Fig. 27. Example of the contents of the TCS-CAIN catalogue, corresponding to the first 20 stars of the field $l=102^{\circ} b=2^{\circ}$.

Table 4. Contents of the TCS-CAIN Catalogue.

\begin{tabular}{|c|c|c|}
\hline 1 & (ID) & ID number of the catalogue \\
\hline 2 & $(\alpha)$ & Right ascension J2000 (degrees) \\
\hline 3 & $(\delta)$ & Declination J2000 (degrees) \\
\hline 4 & (l) & Galactic longitude (degrees) \\
\hline 5 & (b) & Galactic latitude (degrees) \\
\hline 6 & $(x)$ & $X$ position in the frame (pixels) \\
\hline 7 & (y) & $Y$ position in the frame (pixels) \\
\hline 8 & $(J)$ & $J$ magnitude (99.999 means no detection) \\
\hline 9 & $(H)$ & $H$ magnitude (99.999 means no detection) \\
\hline 10 & $(K)$ & $K_{\mathrm{s}}$ magnitude (99.999 means no detection) \\
\hline 11 & $(N)$ & Number of contributing overlapping images \\
\hline 12 & (ID 2 MASS $)$ & Cross number with 2 MASS catalogue \\
\hline
\end{tabular}

magnitudes. The TCS-CAIN data are in good agreement with the predictions, although with a higher dispersion probably due to the larger area used for each 2MASS position, which tends to average out the extinction. Figure 26 also shows the predictions of the model for star counts up to $m_{K}<15$. These are systematically higher than the observed 2MASS counts, as completeness begins to affect the 2MASS survey. The TCS-CAIN, however, still does follow the model counts, and the effect is even more pronounced in the inner disc.

\section{The catalogue}

The digital distribution of the catalogue (named TCS-CAIN Catalogue) will be available via the web. The catalogue contains positions and $J, H, K$ magnitudes for approximately 10 million stars detected at least in one of the three bands of the survey. The main contents of the catalogue are described in Table 4, while Fig. 27 shows an example of the first detections in the field $l=102^{\circ} b=2^{\circ}$. Note that the ID number of the catalogue has the form: "cainlll $+/-b b b$ ffnnnn", where $l l l+/-b b b$ designates the galactic coordinates of the centre of the field of the image, and ffnnnn = frame_number (from 11 to 55$) \times 10000+$ running number. For example, the first star detected in the second frame corresponding to the field $l=26^{\circ} b=-1^{\circ}$ will be named "cain260010-120001". Also note that for the matrix formulation of the transformation from equatorial to Galactic coordinates,

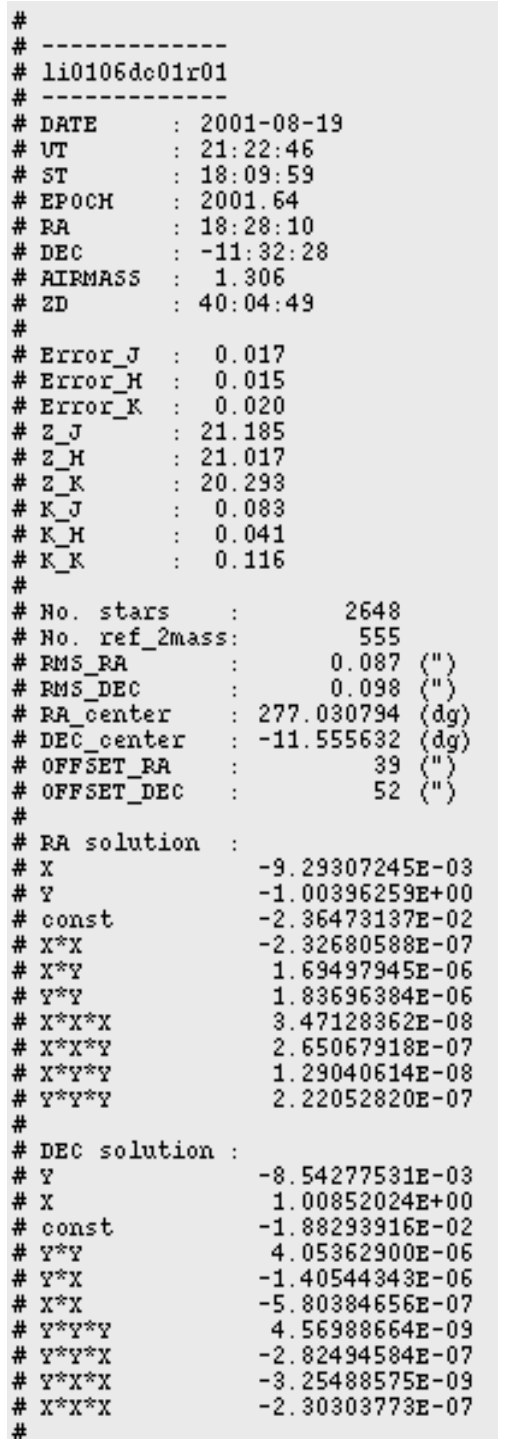

Fig. 28. Example of the contents of the "frame information" of the TCS-CAIN catalogue. This kind of information is available for each of the frames observed in a single field, and it is shown as a header at the beginning of each catalogue file (Fig. 27). 
we used $\alpha_{\mathrm{NGP}}=192.85948^{\circ}, \delta_{\mathrm{NGP}}=27.12825^{\circ}$, and $\theta_{0}=$ $122.93192^{\circ}$ (ESA 1997; Vol. 1).

Along with the catalogue point sources, other information is provided for each frame (the so-called "frame information") including observation date, photometric zero-points, atmospheric extinction coefficients, errors in magnitude, number of measured and reference 2MASS stars in the area of sky covered in the frame, position error, exact tangent point and astrometric coefficients of the cubic solution (which allows the $x, y$ coordinates to be translated in coordinates in the sky) (see an example in Fig. 28). Note that the photometric zero-points, observation date, etc, are the same for the whole field but the astrometric solutions are specific for each frame independently.

The TCS-CAIN Catalogue is now available at www.iac.es/project/tmgs/tcs-cain.html and it will be provided at the CDS Strasbourg data centre very soon. The catalogue has been grouped by its central galactic coordinates, with total of 530 files available. The mean size of the archives is around 2.5 MBytes, but it depends on the number of sources detected (the typical sizes vary from 0.3 MBytes to 6 MBytes in the more crowded fields). The total size of the whole catalogue is $1.1 \mathrm{~GB}$, so it can be easily downloaded. Flags regarding the astrometric accuracy of the frames and the photometric quality of the field (including any significant shift compared to 2MASS photometry) will be addressed on the web server, together with a README file with more specific details of the catalogue generation.

\section{Conclusions}

In this paper we have described the TCS-CAIN survey. This is a deep multi-colour NIR survey of near plane areas of the Milky Way covering a total area of $\sim 41 \mathrm{deg}^{2}$ in the sky. The full point source catalogue derived is now accessible and contains $\sim 10$ million point source detections in $J, H$, and $K_{\mathrm{s}}$ bands with a photometric accuracy of $\sim 0.1 \mathrm{mag}$ in the three bands and a position accuracy of $\sim 0.2^{\prime \prime}$.

The TCS-CAIN survey is at least one magnitude deeper than 2MASS or DENIS, and even more so in the inner Galaxy, as it is less limited by source confusion than those NIR surveys. We have shown that the TCS-CAIN survey has no systematic differences in its photometry or astrometry compared to that of 2MASS. This means that the 2MASS and TCS-CAIN star counts should be directly comparable. Therefore, fainter TCS-CAIN counts can be combined with those of 2MASS to provide a deeper view of the Galaxy.

Acknowledgements. Thanks are given to the anonymous referee, who made a very important suggestion that has improved the overall quality of the catalogue presented here. The author also wants to thank all the people who have participated in the collection of data of the TCS-CAIN survey: M. LópezCorredoira, S. Picaud, N. Castro-Rodríguez, and B. Castro-Rodríguez. The TCS is operated on the island of Tenerife by the Instituto de Astrofísica de Canarias in the Spanish Observatorio del Teide of the Instituto de Astrofísica de Canarias. This publication makes use of data products from the Two Micron All Sky Survey, which is a joint project of the University of Massachusetts and the Infrared Processing and Analysis Center/California Institute of Technology, funded by the National Aeronautics and Space Administration and the National Science Foundation.

\section{References}

Bahcall, J. H. 1986, ARA\&A, 24, 577

Babusiaux, C., \& Gilmore, G. 2005, MNRAS, 358, 1309

Bissantz, N., \& Gerhard, O. E. 2002, MNRAS, 330, 591

Boggess, N. W., Mather, J. C., Weiss, R., et al. 1992, ApJ, 397, 420

Cabrera-Lavers, A., \& Garzón, F. 2003, A\&A, 403, 383

Cabrera-Lavers, A., Garzón, F., \& Hammersley, P. L. 2005, 433, 173

Cutri, R. M., et al. 2003, VizieR Online Data Catalog, 2246, 0

Drimmel, R., Cabrera-Lavers, A., \& López-Corredoira, M. 2003, A\&A, 409, 205

Epchtein, N., Deul, E., Derriere, S., et al. 1999, A\&A, 349, 236

ESA 1997, The Hipparcos and Tycho Catalogues, ESA SP-1200

Freeman, K. C. 1987, ARA\&A, 25, 603

Garzón, F., Hammersley, P. L., Mahoney, T. J., et al. 1993, MNRAS, 264, 773

Gilmore, G. F., Wyse, R. G., \& Kuijken, K. 1989, ARA\&A, 27, 555

Hammersley, P. L., Garzón, F., Mahoney, T. J., \& Calbet, X. 1994, MNRAS, 269, 753

Hammersley, P. L., Garzón, F., Mahoney, T. J., \& Calbet, X. 1995, MNRAS, 273, 206

Hammersley, P. L., Garzón, F., Mahoney, T. J., et al. 2000, MNRAS, 317, L45

Hunt, L. K., Mannucci, F., Testi, L., et al. 1998, AJ, 115, 2594

Kent, S. M., Dame, T. M., \& Fazio, G. 1991, ApJ, 378, 131

Hayakawa, S., Matsumoto, T., Murakami, H., et al. 1981, A\&A, 100, 116

López-Corredoira, M., Hammersley, P. L., Garzón, F., \& Simmoneau, E. 2000, MNRAS, 313, 392

López-Corredoira, M., Hammersley, P. L., Garzón, F., et al. 2001, A\&A, 373, 139

López-Corredoira, M., Cabrera-Lavers, A., Garzón, F., \& Hammersley, P. L. 2002, A\&A, 394, 883 (L02)

López-Corredoira, M., Cabrera-Lavers, A., Garzón, F., \& Gerhard, O. 2004, A\&A, 421, 953

López-Corredoira, M., Cabrera-Lavers, A., \& Gerhard, O. 2005, A\&A, 439, 107 Majewski, S. R. 1993, ARA\&A, 31, 375

Monet, D., Levine, S., Canzian, B., et al. 2003, AJ, 125, 984

Nishiyama, S., Nagata, T., Baba, D., Haba, Y., et al. 2005, ApJ, 621, L105

Paul, E. R. 1993, in The Milky Way Galaxy and Statistical Cosmology, 18901924 (Cambridge: Cambridge University Press)

Picaud, S., Cabrera-Lavers, A., \& Garzón, F. 2003, A\&A, 408, 141

Reid, M. J. 1993, ARA\&A, 31, 345

Robin, A. C., Crézé, M., \& Mohan, V. 1992, ApJ, 400, L25

Robin, A. C., Reylé, C., Derriére, S., \& Picaud, S. 2003, A\&A, 409, 523

Schmidt-Kaler, T. 1977, A\&A, 54, 771

Skrutskie, M. F., Schneider, S. E., Stiening, R., et al. 1997, in The Impact of Large Scale Near-IR Sky Surveys, ed. F. Garzón, N. Epchtein, A. Omont, B. Burton, \& P. Persi (Dordrecht: Kluwer), 25

Stetson, P. B. 1987, PASP, 99, 191

Wainscoat, R. J., Cohen, M., Volk, K., Walzer, H. J., \& Schwartz, D. E. 1992, ApJS, 83, 111

Zacharias, N., Urban, S., Zacharias, M., et al. 2004, AJ, 127, 3043 